hep-ph/0302021

IFIC/03-03

ZU-TH02/03

\title{
Solar Neutrino Masses and Mixing from Bilinear R-Parity Broken Supersymmetry: Analytical versus Numerical Results
}

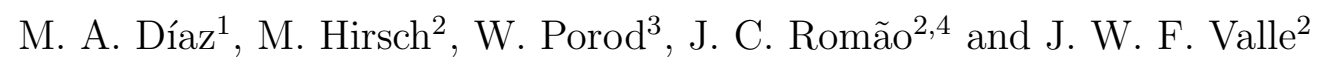 \\ ${ }^{1}$ Facultad de Física, Universidad Católica de Chile \\ Av. Vicuña Mackenna 4860, Santiago, Chile \\ ${ }^{2}$ AHEP Group, Instituto de Física Corpuscular - C.S.I.C./Universitat de València \\ Edificio de Institutos de Paterna, Apartado 22085, E-46071 València, Spain \\ ${ }^{3}$ Institut für Theoretische Physik, Universität Zürich, \\ CH-8057 Zürich, Switzerland \\ ${ }^{4}$ Departamento de Física and CFIF, Instituto Superior Técnico \\ Av. Rovisco Pais 1, 1049-001 Lisboa, Portugal
}

\begin{abstract}
We give an analytical calculation of solar neutrino masses and mixing at one-loop order within bilinear R-parity breaking supersymmetry, and compare our results to the exact numerical calculation. Our method is based on a systematic perturbative expansion of R-parity violating vertices to leading order. We find in general quite good agreement between approximate and full numerical calculation, but the approximate expressions are much simpler to implement. Our formalism works especially well for the case of the large mixing angle MSW solution (LMA-MSW), now strongly favoured by the recent KamLAND reactor neutrino data.
\end{abstract}




\section{Introduction}

Solar neutrino experiments, including the measurement of the neutral current rate for solar neutrinos by the SNO collaboration 1] provide a solid evidence for solar neutrino conversions 2]. This has been recently confirmed by the first results from the KamLAND experiment using reactor (anti)-neutrinos [3, 4]. Combining the information from reactors with all of the solar neutrino data leads to the best fit point [5]:

$$
\tan ^{2} \theta_{\mathrm{SOL}}=0.46, \quad \Delta m_{\mathrm{SOL}}^{2}=6.9 \times 10^{-5} \mathrm{eV}^{2},
$$

confirming that the solar neutrino mixing angle is large, but significantly non-maximal. The $3 \sigma$ region for $\theta$ is:

$$
0.29 \leq \tan ^{2} \theta_{\mathrm{SOL}} \leq 0.86
$$

based on a combination of all experimental data. However, one finds a significant reduction of the allowed $\Delta m_{\mathrm{SOL}}^{2}$ range. As shown in Ref. [5], the pre-KamLAND LMA-MSW region is now split into two sub-regions. At $3 \sigma$ (1 dof.) one obtains

$$
5.1 \times 10^{-5} \mathrm{eV}^{2} \leq \Delta \mathrm{m}_{\mathrm{SOL}}^{2} \leq 9.7 \times 10^{-5} \mathrm{eV}^{2}, 1.2 \times 10^{-4} \mathrm{eV}^{2} \leq \Delta \mathrm{m}_{\mathrm{SOL}}^{2} \leq 1.9 \times 10^{-4} \mathrm{eV}^{2}
$$

Altogether, KamLAND results exclude all oscillation solutions except for the large mixing angle MSW solution (LMA-MSW) to the solar neutrino problem [6].

On the other hand, current atmospheric neutrino data require oscillations involving $\nu_{\mu} \leftrightarrow \nu_{\tau}$ [7]. The most recent global analysis gives [2],

$$
\sin ^{2} \theta_{\mathrm{ATM}}=0.5, \Delta m_{\mathrm{ATM}}^{2}=2.5 \times 10^{-3} \mathrm{eV}^{2}
$$

with the $3 \sigma$ ranges ( 1 d.o.f.)

$$
\begin{array}{r}
0.3 \leq \sin ^{2} \theta_{\text {ATM }} \leq 0.7 \\
1.2 \times 10^{-3} \mathrm{eV}^{2} \leq \Delta \mathrm{m}_{\text {ATM }}^{2} \leq 4.8 \times 10^{-3} \mathrm{eV}^{2} .
\end{array}
$$

These data have triggered a rush of theoretical and phenomenological papers on models of neutrino masses and mixings, most of which introduce a large mass scale in order to 
implement various variants of the see-saw mechanism [8, 9, 10]. Broken R-parity supersymmetry provides a theoretically interesting and phenomenologically viable alternative to the origin of neutrino mass and mixing [11]. Here we focus on the simplest case of supersymmetry with bilinear R-parity breaking [12]. In contrast to the seesaw mechanism, here neutrino masses are generated at the electro-weak scale. Such low-scale schemes for neutrino masses have the advantage of being testable also in accelerator experiments [13]17] through the decay properties of the lightest supersymmetric particle if the LSP is a neutralino [14, 15, 16], a slepton [17] or a stop [18, 19].

Supersymmetric models with explicit bilinear breaking of R-parity (BRpV) [20, 21, 22, 23, 24, 25, 26, 27] provide a simple and calculable framework for neutrino masses and mixing angles in agreement with the experimental data [28]. In this model the atmospheric neutrino mass scale is generated at tree-level, through an effective "low-scale" variant of the seesaw mechanism [11]. In contrast, the solar mass and mixings are generated radiatively [28]. Tree-level neutrino masses within BRpV have been treated extensively in the literature.

This paper is mainly devoted to the solar neutrino masses and mixing. An accurate and reliable calculational method is now necessary in order to confront the model with the new experimental data from KamLAND and other neutrino experiments. A complete one-loop calculation of the neutrino-neutralino mass matrix has been given [28] but is rather complex. On the other hand, approximations to the full 1-loop calculation which exist in the literature [29] have not been tested yet against the full calculation. Especially in view of future experimental sensitivities we think such a "benchmark" is important.

In this paper we give an accurate determination of neutrino mass and mixing within an analytical approximation and obtain formulae which can be rather simple, in some cases. For definiteness we will stick to the case of explicit BRpV only. This is the simplest of all R parity violating models. It can be considered either as a minimal three-parameter extension of the MSSM (with no new particles) valid up to some very high unification energy scale, or as the effective description of a more fundamental theory in which the breaking of R-parity is spontaneous [30, 31, 32]. The latter implies the absence of trilinear 
R-parity breaking parameters in the superpotential ${ }^{1}$.

This paper is organized as follows. In Sec. 2 we introduce the main features of the model and the relevant mass matrices and corresponding diagonalization matrices. In particular we identify the relevant Feynman graph topologies and rules, and derive approximate formulae for the couplings relevant for the determination of radiatively induced solar neutrino mass scale. We give approximate formulas for the bottom quark/squark loop as well as for the charged scalar loop. In Sec. 3 we check the accuracy of our approximation formulas by a comparison with a full numerical calculation, studying first the role of the simplest bottom-sbottom loop, then the charged scalar loop, before comparing the sum of the two to the full numerical result. In 4 we give simplified approximation formulas for the solar mass and solar mixing angle and conclude and summarize our results in 5 .

\section{BRpV Formalism}

In this section we introduce the main features of the model and the relevant mass matrices, and develop approximate formulas, first for couplings and then for the radiative contributions to the neutrino masses due to the exchange of bottom and sbottom quarks, and due to charged scalars and charged fermion loops.

\section{$2.1 \quad$ BRpV Model}

The minimal BRpV model we are working with is characterized by the presence of three extra bilinear terms in the superpotential analogous to the $\mu$ term present in the MSSM

$$
W=W_{Y u k}+\varepsilon_{a b}\left(-\mu \widehat{H}_{d}^{a} \widehat{H}_{u}^{b}+\epsilon_{i} \widehat{L}_{i}^{a} \widehat{H}_{u}^{b}\right)
$$

where $W_{Y u k}$ includes the usual MSSM Yukawa terms, $\mu$ is the Higgsino mass term of the MSSM, and $\epsilon_{i}$ are the three new terms which violate R-Parity and lepton number. The smallness of $\epsilon_{i}$ may arise dynamically (the product of a Yukawa coupling times a singlet sneutrino vacuum expectation value) in models with spontaneous breaking of $\mathrm{R}$ parity [30].

\footnotetext{
${ }^{1}$ Alternatively, such absence may arise from suitable symmetries 33 ]
} 
Alternatively, the smallness of the $\epsilon_{i}$ may arise from suitable family symmetries 33. In fact any solution to the $\mu$ problem [34] potentially explains also the " $\epsilon_{i}$-problem" [35]. In fact a common origin for the $\epsilon_{i}$ terms responsible for the explanation of the neutrino anomalies, and the $\mu$ term accounting for electroweak symmetry breaking can be ascribed to a suitable horizontal symmetry that may also predict their ratio, as in 33].

In addition we have the corresponding soft supersymmetry breaking terms in the scalar potential,

$$
V_{\text {soft }}=V_{\text {soft }}^{\prime}+\varepsilon_{a b}\left(-B \mu H_{d}^{a} H_{u}^{b}+B_{i} \epsilon_{i} \widetilde{L}_{i}^{a} H_{u}^{b}\right)
$$

where $B$ and the three $B_{i}$ have units of mass and in $V_{\text {soft }}^{\prime}$ we include all the usual mass and trilinear supersymmetry breaking terms of the MSSM.

\subsection{Rotation Matrices}

If the effective $\mathrm{RpV}$ parameters are smaller than the weak scale, we can work in a perturbative expansion defined by $\xi \ll 1$, where $\xi$ denotes a $3 \times 4$ matrix given as [36]

$$
\begin{aligned}
\xi_{i 1} & =\frac{g^{\prime} M_{2} \mu}{2 \Delta_{0}} \Lambda_{i} \\
\xi_{i 2} & =-\frac{g M_{1} \mu}{2 \Delta_{0}} \Lambda_{i} \\
\xi_{i 3} & =-\frac{\epsilon_{i}}{\mu}+\frac{M_{\tilde{\gamma}} v_{u}}{4 \Delta_{0}} \Lambda_{i} \\
\xi_{i 4} & =-\frac{M_{\tilde{\gamma}} v_{d}}{4 \Delta_{0}} \Lambda_{i}
\end{aligned}
$$

where $\Delta_{0}$ is the determinant of the $4 \times 4$ neutralino mass matrix, $M_{\tilde{\gamma}}=g^{2} M_{1}+g^{\prime 2} M_{2}$ and

$$
\Lambda_{i}=\mu v_{i}+v_{d} \epsilon_{i}
$$

The neutralino/neutrino mass matrix is diagonalized by a $7 \times 7$ rotation matrix $\mathcal{N}$ according to

$$
\mathcal{N}^{*} \mathbf{M}_{F^{0}} \mathcal{N}^{-1}=\mathbf{M}_{F 0}^{\text {diag }}
$$


and the eigenvectors are given by

$$
F_{i}^{0}=\mathcal{N}_{i j} \psi_{j}
$$

using the basis $\psi=\left(-i \lambda^{\prime},-i \lambda^{3}, \widetilde{H}_{d}^{1}, \widetilde{H}_{u}^{2}, \nu_{e}, \nu_{\mu}, \nu_{\tau}\right)$. In this approximation, the rotation matrix can be written as

$$
\mathcal{N}^{*} \approx\left(\begin{array}{cc}
N^{*} & N^{*} \xi^{\dagger} \\
-V_{\nu}^{T} \xi & V_{\nu}^{T}
\end{array}\right)
$$

Here, $N$ is the rotation matrix that diagonalizes the $4 \times 4$ MSSM neutralino mass matrix, $V_{\nu}$ is the rotation matrix that diagonalizes the tree level neutrino $3 \times 3$ mass matrix, and $\xi_{i j} \ll 1$ are the expansion parameters [36, 37]. The terms we need are

$$
V_{\nu}^{T} \xi=\left(\begin{array}{cccc}
0 & 0 & b \tilde{\epsilon}_{1} & 0 \\
0 & 0 & b \tilde{\epsilon}_{2} & 0 \\
a_{1}|\vec{\Lambda}| & a_{2}|\vec{\Lambda}| & a_{3}|\vec{\Lambda}|+b \tilde{\epsilon}_{3} & a_{4}|\vec{\Lambda}|
\end{array}\right)
$$

where $b=-1 / \mu$,

$$
a_{1}=\frac{g^{\prime} M_{2} \mu}{2 \Delta_{0}}, \quad a_{2}=-\frac{g M_{1} \mu}{2 \Delta_{0}}, \quad a_{3}=\frac{M_{\tilde{\gamma}} v_{u}}{4 \Delta_{0}}, \quad a_{4}=-\frac{M_{\tilde{\gamma}} v_{d}}{4 \Delta_{0}}
$$

The $\tilde{\epsilon}$ parameters in eq. (14) are defined as $\tilde{\epsilon}_{i}=\left(V_{\nu}^{T}\right)^{i j} \epsilon_{j}$, and are given by

$$
\begin{aligned}
\tilde{\epsilon}_{1} & =\frac{\epsilon_{e}\left(\Lambda_{\mu}^{2}+\Lambda_{\tau}^{2}\right)-\Lambda_{e}\left(\Lambda_{\mu} \epsilon_{\mu}+\Lambda_{\tau} \epsilon_{\tau}\right)}{\sqrt{\Lambda_{\mu}^{2}+\Lambda_{\tau}^{2}} \sqrt{\Lambda_{e}^{2}+\Lambda_{\mu}^{2}+\Lambda_{\tau}^{2}}} \\
\tilde{\epsilon}_{2} & =\frac{\Lambda_{\tau} \epsilon_{\mu}-\Lambda_{\mu} \epsilon_{\tau}}{\sqrt{\Lambda_{\mu}^{2}+\Lambda_{\tau}^{2}}} \\
\tilde{\epsilon}_{3} & =\frac{\vec{\Lambda} \cdot \vec{\epsilon}}{\sqrt{\Lambda_{e}^{2}+\Lambda_{\mu}^{2}+\Lambda_{\tau}^{2}}}
\end{aligned}
$$

On the other hand the chargino/charged slepton mass matrix is diagonalized with two different $5 \times 5$ mass matrices,

$$
\mathcal{U}^{*} \mathbf{M}_{F^{+}} \mathcal{V}^{-1}=\mathbf{M}_{F^{+}}^{\text {diag }}
$$

with the eigenvectors satisfying

$$
F_{R i}^{+}=\mathcal{V}_{i j} \psi_{j}^{+}, \quad F_{L i}^{-}=\mathcal{U}_{i j} \psi_{j}^{-}
$$


in the basis $\psi^{+}=\left(-i \lambda^{+}, \widetilde{H}_{2}^{1}, e_{R}^{+}, \mu_{R}^{+}, \tau_{R}^{+}\right)$and $\psi^{-}=\left(-i \lambda^{-}, \widetilde{H}_{1}^{2}, e_{L}^{-}, \mu_{L}^{-}, \tau_{L}^{-}\right)$, and with the Dirac fermions being

$$
F_{i}^{+}=\left(\begin{array}{l}
F_{R i}^{+} \\
\frac{F_{L i}^{-}}{}
\end{array}\right)
$$

To first order in the R-Parity violating parameters we have

$$
\mathcal{V} \approx\left(\begin{array}{cc}
V & V \xi_{R}^{T} \\
-V_{R}^{\ell} \xi_{R}^{*} & V_{R}^{\ell}
\end{array}\right), \quad \mathcal{U} \approx\left(\begin{array}{cc}
U & U \xi_{L}^{\dagger} \\
-V_{L}^{\ell *} \xi_{L} & V_{L}^{\ell *}
\end{array}\right)
$$

where $V_{L}^{\ell *}$ and $V_{R}^{\ell}$ diagonalize the charged lepton mass matrix according to $V_{L}^{\ell *} \mathbf{M}^{\ell} V_{R}^{\ell \dagger}=$ $\mathbf{M}_{\mathrm{d} i a g}^{\ell}$. For the purposes of our approximate formula, it is sufficient to take $\xi_{R}=\mathbf{0}_{2 \times 3}$, because the mixing between right-handed leptons and the charginos is supressed with respect to $\xi_{L}$ by a factor of $m_{l} / M_{S U S Y}\left[36\right.$, 37]. Note, that we can choose $V_{L}^{\ell *}=V_{R}^{\ell \dagger}=\mathbf{1}_{3 \times 3}$. We then have

$$
\xi_{L}^{i 1}=a_{1}^{L} \Lambda_{i}, \quad \xi_{L}^{i 2}=a_{2}^{L} \Lambda_{i}+b \epsilon_{i}
$$

and

$$
a_{1}^{L}=\frac{g}{\sqrt{2} \Delta_{+}}, \quad a_{2}^{L}=-\frac{g^{2} v_{u}}{2 \mu \Delta_{+}}
$$

where $\Delta_{+}$is the determinant of the $2 \times 2$ chargino mass matrix.

In the BRpV model the charged Higgs fields mix with the charged sleptons forming an $8 \times 8$ mass matrix [28], which is diagonalized by a rotation matrix $\mathbf{R}_{S^{ \pm}}$. The construction of $\mathbf{R}_{S^{ \pm}}$to first order in small ( $\mathrm{RpV}$ ) parameters is quite straightforward but lengthy. The interested reader can find the details in Appendix A.

\subsection{Approximate Couplings}

The relevant Feynman rules for the bottom-sbottom loops are, in the case of left sbottoms:

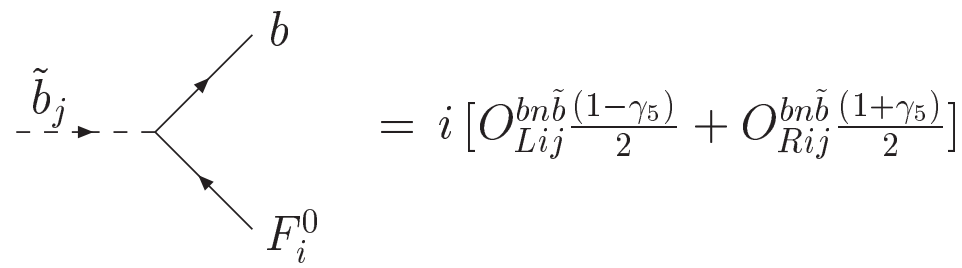


with

$$
\begin{aligned}
O_{L i j}^{b n \tilde{b}} & =-R_{j 1}^{\tilde{b}} h_{b} \mathcal{N}_{i 3}^{*}-R_{j 2}^{\tilde{b}} \frac{2 g}{3 \sqrt{2}} t_{W} \mathcal{N}_{i 1}^{*} \\
O_{R i j}^{b n \tilde{b}} & =R_{j 1}^{\tilde{b}} \frac{g}{\sqrt{2}}\left(\mathcal{N}_{i 2}-\frac{1}{3} t_{W} \mathcal{N}_{i 1}\right)-R_{j 2}^{\tilde{b}} h_{b} \mathcal{N}_{i 3}^{*}
\end{aligned}
$$

where $t_{W}=\tan \theta_{W}$. After approximating the rotation matrix $\mathcal{N}$ we find that expressions similar to eq. (23) with the replacement $\mathcal{N} \rightarrow \mathrm{N}$ are valid when the neutral fermion is a neutralino. When the neutral fermion $F^{0}$ is a neutrino, the following expressions hold

$$
\begin{aligned}
O_{L i j}^{b n \tilde{b}} & \approx R_{j 1}^{\tilde{b}} h_{b}\left(a_{3}|\vec{\Lambda}| \delta_{i^{\prime} 3}+b \tilde{\epsilon}_{i^{\prime}}\right)+R_{j 2}^{\tilde{b}} \frac{2 g}{3 \sqrt{2}} t_{W} a_{1}|\vec{\Lambda}| \delta_{i^{\prime} 3} \\
O_{R i j}^{b n \tilde{b}} & \approx R_{j 1}^{\tilde{b}} \frac{g}{\sqrt{2}}\left(\frac{1}{3} t_{W} a_{1}-a_{2}\right)|\vec{\Lambda}| \delta_{i^{\prime} 3}+R_{j 2}^{\tilde{b}} h_{b}\left(a_{3}|\vec{\Lambda}| \delta_{i^{\prime} 3}+b \tilde{\epsilon}_{i^{\prime}}\right)
\end{aligned}
$$

where $i^{\prime}=i-4$ label one of the neutrinos. $R_{j k}^{\tilde{b}}$ are the rotation matrices connecting weak and mass eigenstate basis for the scalar bottom quarks. In case of no intergenerational mixing in the squark sector $R_{j k}^{\tilde{b}}$ can be parameterized by just one diagonalizing angle $\theta_{\tilde{b}}$.

The relevant Feynman rule for the charged Higgs/slepton loops is

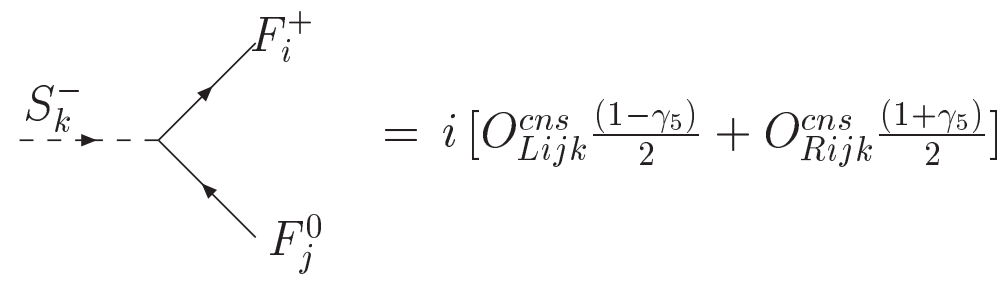

where the $O_{\text {Lijk }}^{c n s}$ and $O_{\text {Rijk }}^{c n s}$ couplings are given in Appendix B] in Eqs. (56, 57).

After approximating the rotation matrices $\mathcal{U}$, and $\mathcal{V}$ in the chargino sector, and $\mathcal{N}$ in the neutralino sector we find approximate expressions for these couplings that we will use below. These formulae are collected in Eqs. (57, 58, 59, 60) of Appendix [B,

\subsection{Relevant Topologies}

We now give the structure of the mass matrices relevant for the determination of solar neutrino masses and mixings. While in the $\mathrm{BRpV}$ model the atmospheric anomaly is explained at the tree-level, the solar neutrino masses and mixings are both generated radiatively. In particular, the "solar angle" has no meaning at the tree level due to the degeneracy of the two lightest neutrinos in this limit. 
Diagonalizing the tree-level neutrino mass matrix first and adding then the 1-loop corrections before re-diagonalization the resulting neutrino/neutralino mass matrix has nonzero entries in the neutrino/neutrino, the neutrino/neutralino and in the neutralino/neutralino sectors. We have found that the most important part of the 1-loop neutrino masses derives from the neutrino/neutrino sector and that the 1-loop induced neutrino/neutralino mixing is usually subdominant.

The relevant topologies for the one loop calculation of neutrino masses are then illustrated in Fig 1. Here our conventions are as follows: open circles with a cross inside

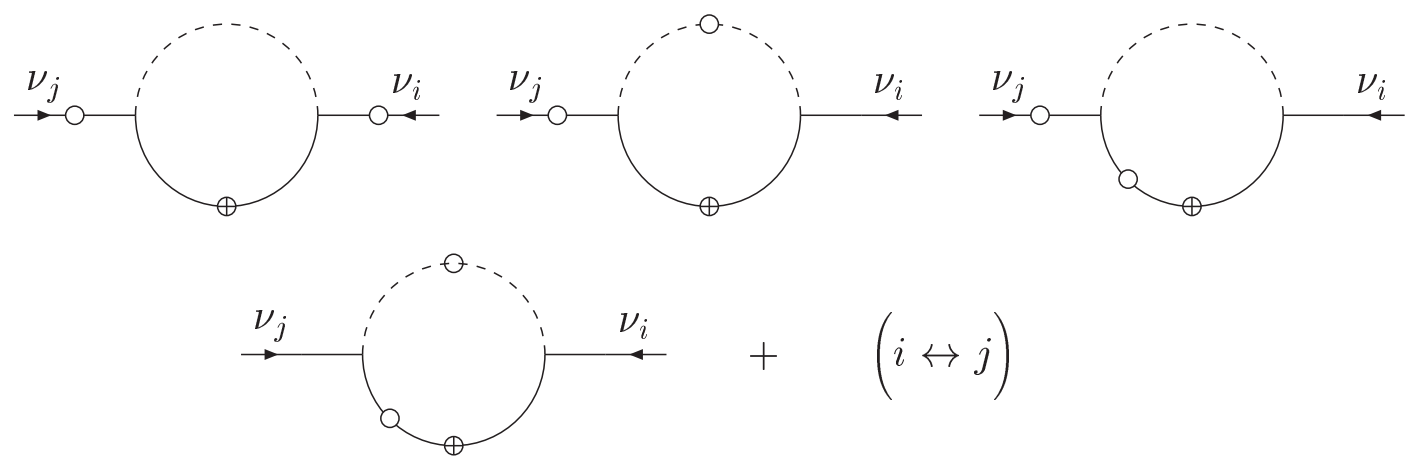

Figure 1: Topologies for neutrino self-energies in the BRpV supersymmetric model

indicate genuine mass insertions which flip chirality. On the other hand open circles without a cross correspond to small R-Parity violating projections, indicating how much of an Rp-even/odd mass eigenstate is present in a given Rp-odd/even weak eigenstate. Strictly speaking these projections are really coupling matrices attached to the vertices, and this is what appears in the numerical code. However, given the smallness of Rp-violating effects, the "insertion-method" proves to be a rather useful tool to develop an analytical perturbative expansion and to acquire some simple understanding of the results.

\subsection{Bottom-sbottom loops}

The simplest contribution to the radiatively induced neutrino mass arises from loops involving bottom quarks and squarks is given by [28]

$$
\widetilde{\Pi}_{i j}(0)=-\frac{N_{c}}{16 \pi^{2}} \sum_{r}\left(O_{R j r}^{b n \tilde{b}} O_{L i r}^{b n \tilde{b}}+O_{L j r}^{b n \tilde{b}} O_{R i r}^{b n \tilde{b}}\right) m_{b} B_{0}\left(0, m_{b}^{2}, m_{r}^{2}\right)
$$


$B_{0}\left(0, m_{b}^{2}, m_{r}^{2}\right)$ is the usual Passarino-Veltman function [38, 39]. This contribution can be expressed as being proportional to the difference of two $B_{0}$ functions,

$$
\Delta B_{0}^{\tilde{b}_{1} \tilde{b}_{2}}=B_{0}\left(0, m_{b}^{2}, m_{\tilde{b}_{1}}^{2}\right)-B_{0}\left(0, m_{b}^{2}, m_{\tilde{b}_{2}}^{2}\right)
$$

as follows

$$
\Delta \widetilde{\Pi}_{i j}=-\frac{N_{c} m_{b}}{16 \pi^{2}} 2 s_{\tilde{b}} c_{\tilde{b}} h_{b}^{2} \Delta B_{0}^{\tilde{b}_{1} \tilde{b}_{2}}\left[\frac{\tilde{\epsilon}_{i} \tilde{\epsilon}_{j}}{\mu^{2}}+a_{3} b\left(\tilde{\epsilon}_{i} \delta_{j 3}+\tilde{\epsilon}_{j} \delta_{i 3}\right)|\vec{\Lambda}|+\left(a_{3}^{2}+\frac{a_{L} a_{R}}{h_{b}^{2}}\right) \delta_{i 3} \delta_{j 3}|\vec{\Lambda}|^{2}\right]
$$

where we have defined

$$
a_{R}=\frac{g}{\sqrt{2}}\left(\frac{1}{3} t_{W} a_{1}-a_{2}\right), \quad a_{L}=\frac{g}{\sqrt{2}} \frac{2}{3} t_{W} a_{1}
$$

The different contributions can be understood as coming from the graphs corresponding to the first topology of Fig. 1. They have been depicted in more detail in Fig. 2, where

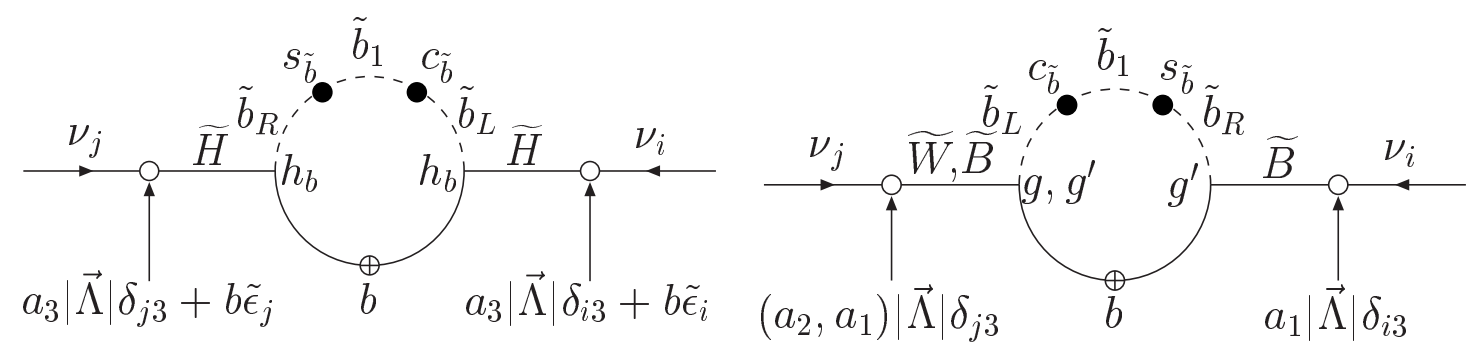

Figure 2: Bottom-Sbottom diagrams for solar neutrino mass in the BRpV model

we have adopted the following conventions: a) as before, open circles correspond to small R-parity violating projections, indicating how much of a weak eigenstate is present in a given mass eigenstate, (b) full circles correspond to R-parity conserving projections and (c) open circles with a cross inside indicate genuine mass insertions which flip chirality.

The open and full circles should really appear at the vertices since the particles propagating in the loop are the mass eigenstates. We have however separated them to better identify the origin of the various terms. There is another set of graphs analogous to the previous ones which corresponds to the heavy sbottom. They are obtained from the previous graphs making the replacement $\tilde{b}_{1} \rightarrow \tilde{b}_{2}, s_{\tilde{b}} \rightarrow c_{\tilde{b}}$ and $c_{\tilde{b}} \rightarrow-s_{\tilde{b}}$. Note that for all contributions to the $2 \times 2$ submatrix corresponding to the light neutrinos the divergence from $B_{0}\left(0, m_{b}^{2}, m_{\tilde{b}_{1}}^{2}\right)$ is canceled by the divergence from $B_{0}\left(0, m_{b}^{2}, m_{\tilde{b}_{2}}^{2}\right)$, making finite the 
contribution from bottom-sbottom loops to this submatrix, as it should be, since the mass is fully "calculable".

\subsection{Charged Scalar-Charged Fermion Loops}

Another contribution to the radiatively induced neutrino mass comes from chargedscalar/charged-fermion loops, given as [28]

$$
\widetilde{\Pi}_{i j}(0)=-\frac{1}{16 \pi^{2}} \sum_{k, r}\left(O_{R k j r}^{c n s} O_{L k i r}^{c n s}+O_{L k j r}^{c n s} O_{R k i r}^{c n s}\right) m_{k} B_{0}\left(0, m_{k}^{2}, m_{r}^{2}\right)
$$

The structure of the contribution from charged Higgs/slepton loops is substantially more complex than that of the bottom-sbottom loop considered above. It can be expressed as

$$
\begin{aligned}
\Delta \widetilde{\Pi}_{i j}=\frac{m_{\tau}}{16 \pi^{2}}[ & C_{i j}^{\tilde{\tau}_{2} \tilde{\tau}_{1}} \Delta B_{0}^{\tilde{\tau}_{2} \tilde{\tau}_{1}}+C_{i j}^{H^{ \pm} \tilde{\tau}_{1}} \Delta B_{0}^{H^{ \pm} \tilde{\tau}_{1}}+C_{i j}^{H^{ \pm} \tilde{\tau}_{2}} \Delta B_{0}^{H^{ \pm} \tilde{\tau}_{2}} \\
& +C_{i j}^{H^{ \pm} \tilde{L}_{1}} \Delta B_{0}^{H^{ \pm} L_{1}}+C_{i j}^{H^{ \pm} \tilde{L}_{2}} \Delta B_{0}^{H^{ \pm} L_{2}}+C_{i j}^{G^{ \pm} \tilde{L}_{1}} \Delta B_{0}^{G^{ \pm} L_{1}} \\
& +C_{i j}^{G^{ \pm} \tilde{L}_{2}} \Delta B_{0}^{G^{ \pm} L_{2}}+C_{i j}^{G^{ \pm} \tilde{\tau}_{1} \tilde{\tau}_{2}} \Delta B_{0}^{G^{ \pm} \tilde{\tau}_{1} \tilde{\tau}_{2}}+C_{i j}^{G^{ \pm} H^{ \pm} \tilde{\tau}_{1} \tilde{\tau}_{2}} \Delta B_{0}^{G^{ \pm} H^{ \pm} \tilde{\tau}_{1} \tilde{\tau}_{2}} \\
& +(i \leftrightarrow j)]
\end{aligned}
$$

where

$$
\begin{gathered}
\Delta B_{0}^{X Y} \equiv B_{0}\left(0, m_{\tau}^{2}, m_{X}^{2}\right)-B_{0}\left(0, m_{\tau}^{2}, m_{Y}^{2}\right) ; X, Y=\left(G^{ \pm}, H^{ \pm}, L_{1}, L_{2}, \tilde{\tau}_{1}, \tilde{\tau}_{2}\right) \\
\Delta B_{0}^{G^{ \pm} \tilde{\tau}_{1} \tilde{\tau}_{2}} \equiv c_{\tilde{\tau}}^{2} B_{0}\left(0, m_{\tau}^{2}, m_{\tilde{\tau}_{1}}^{2}\right)+s_{\tilde{\tau}}^{2} B_{0}\left(0, m_{\tau}^{2}, m_{\tilde{\tau}_{2}}^{2}\right)-B_{0}\left(0, m_{\tau}^{2}, m_{G^{ \pm}}^{2}\right) \\
\Delta B_{0}^{G^{ \pm} H^{ \pm} \tilde{\tau}_{1} \tilde{\tau}_{2}} \equiv c_{\beta}^{2} B_{0}\left(0, m_{\tau}^{2}, m_{G^{ \pm}}^{2}\right)+s_{\beta}^{2} B_{0}\left(0, m_{\tau}^{2}, m_{H^{ \pm}}^{2}\right) \\
-c_{\tilde{\tau}}^{2} B_{0}\left(0, m_{\tau}^{2}, m_{\tilde{\tau}_{1}}^{2}\right)-s_{\tilde{\tau}}^{2} B_{0}\left(0, m_{\tau}^{2}, m_{\tilde{\tau}_{2}}^{2}\right)
\end{gathered}
$$

and

$$
\begin{aligned}
& C_{i j}^{\tilde{\tau}_{2} \tilde{\tau}_{1}}=s_{\tilde{\tau}} c_{\tilde{\tau}}\left\{\sqrt{2} g^{\prime} a_{1}|\vec{\Lambda}|\left[g V_{\nu, j 3}^{T} a_{1}^{L} \Lambda_{3}-\frac{1}{\sqrt{2}}\left(g a_{2}+g^{\prime} a_{1}\right)|\vec{\Lambda}| \delta_{j 3}\right] \delta_{i 3}\right. \\
& \left.+h_{\tau}^{2}\left(b \tilde{\epsilon}_{i}+a_{3}|\vec{\Lambda}| \delta_{i 3}-c_{\beta} \frac{v_{3}}{v} V_{\nu, i 3}^{T}\right)\left[b \tilde{\epsilon}_{j}+a_{3}|\vec{\Lambda}| \delta_{j 3}-V_{\nu, j 3}^{T}\left(a_{2}^{L} \Lambda_{3}+b \epsilon_{3}\right)\right]\right\} \\
& C_{i j}^{H^{ \pm} \tilde{\tau}_{1}}=-s_{\beta} \Theta_{H L_{3}}\left\{c_{\tilde{\tau}} h_{\tau} V_{\nu, i 3}^{T}\left[g V_{\nu, j 3}^{T} a_{1}^{L} \Lambda_{3}-\frac{1}{\sqrt{2}}\left(g a_{2}+g^{\prime} a_{1}\right)|\vec{\Lambda}| \delta_{j 3}\right]\right. \\
& \left.+s_{\tilde{\tau}} h_{\tau}^{2} V_{\nu, i 3}^{T}\left[b \tilde{\epsilon}_{j}+a_{3}|\vec{\Lambda}| \delta_{j 3}-V_{\nu, j 3}^{T}\left(a_{2}^{L} \Lambda_{3}+b \epsilon_{3}\right)\right]\right\} \\
& C_{i j}^{H^{ \pm} \tilde{\tau}_{2}}=s_{\beta} \Theta_{H R_{3}}\left\{s_{\tilde{\tau}} h_{\tau} V_{\nu, i 3}^{T}\left[g V_{\nu, j 3}^{T} a_{1}^{L} \Lambda_{3}-\frac{1}{\sqrt{2}}\left(g a_{2}+g^{\prime} a_{1}\right)|\vec{\Lambda}| \delta_{j 3}\right]\right.
\end{aligned}
$$



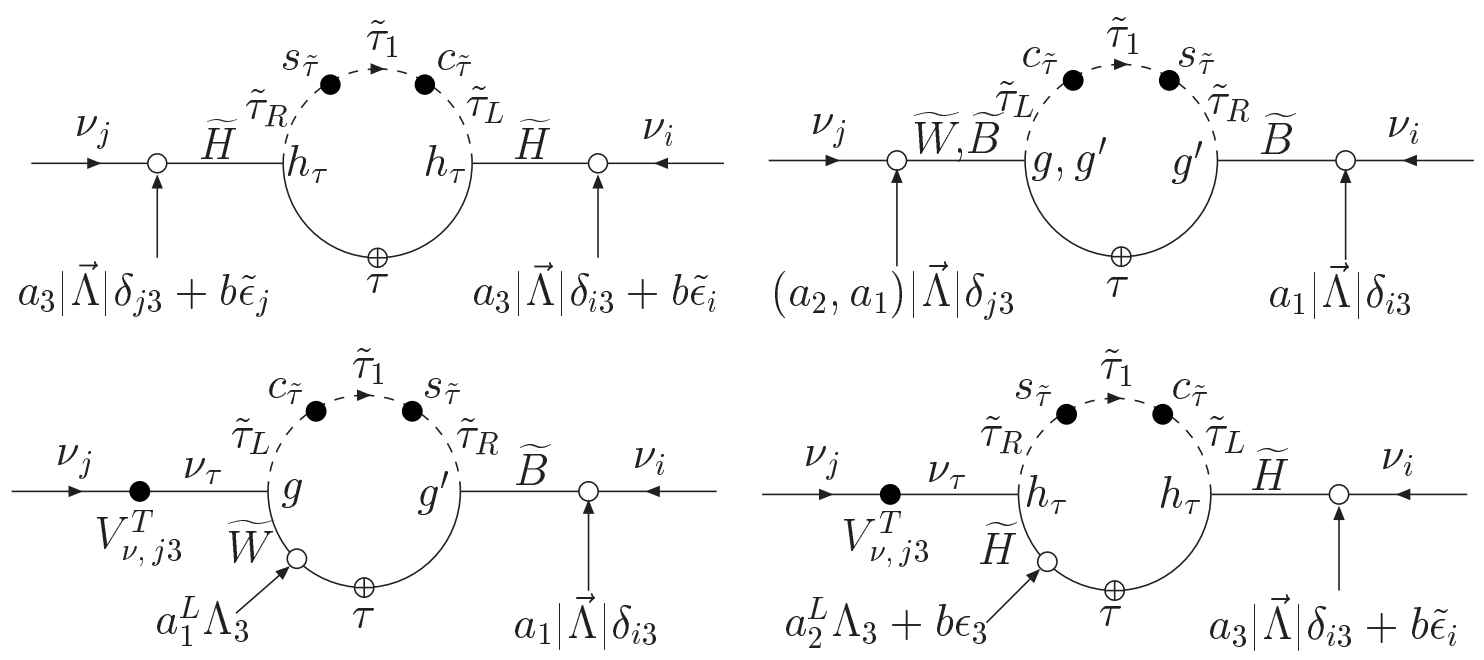

Figure 3: Charged scalar contributions to solar neutrino masses in BRpV model: terms proportional to $\Delta B_{0}^{\tilde{\tau}_{2} \tilde{\tau}_{1}}$.

$$
\begin{aligned}
& \left.\quad-c_{\tilde{\tau}} h_{\tau}^{2} V_{\nu, i 3}^{T}\left[b \tilde{\epsilon}_{j}+a_{3}|\vec{\Lambda}| \delta_{j 3}-V_{\nu, j 3}^{T}\left(a_{2}^{L} \Lambda_{3}+b \epsilon_{3}\right)\right]\right\} \\
& C_{i j}^{H^{ \pm} L_{1}}=-s_{\beta} \widetilde{\Theta}_{H L_{1}} h_{\tau} g V_{\nu, i 3}^{T} V_{\nu, j 1}^{T} a_{1}^{L} \Lambda_{3} \\
& C_{i j}^{H^{ \pm} L_{2}}=-s_{\beta} \widetilde{\Theta}_{H L_{2}} h_{\tau} g V_{\nu, i 3}^{T} V_{\nu, j 2}^{T} a_{1}^{L} \Lambda_{3} \\
& C_{i j}^{G^{ \pm} L_{1}}=-c_{\beta} \frac{v_{1}}{v} h_{\tau} g V_{\nu, i 3}^{T} V_{\nu, j 1}^{T} a_{1}^{L} \Lambda_{3} \\
& C_{i j}^{G^{ \pm} L_{2}}=-c_{\beta} \frac{v_{2}}{v} h_{\tau} g V_{\nu, i 3}^{T} V_{\nu, j 2}^{T} a_{1}^{L} \Lambda_{3} \\
& C_{i j}^{G^{ \pm} \tilde{\tau}_{1} \tilde{\tau}_{2}}=c_{\beta} \frac{v_{3}}{v} h_{\tau} V_{\nu, i 3}^{T}\left[g V_{\nu, j 3}^{T} a_{1}^{L} \Lambda_{3}-\frac{1}{\sqrt{2}}\left(g a_{2}+g^{\prime} a_{1}\right)|\vec{\Lambda}| \delta_{j 3}\right] \\
& C_{i j}^{G^{ \pm} H^{ \pm} \tilde{\tau}_{1} \tilde{\tau}_{2}}=h_{\tau} g \tilde{\epsilon}_{i} V_{\nu, i 3}^{T} b a_{1}^{L} \Lambda_{3}
\end{aligned}
$$

The result of Eq. (30) can be represented graphically for better understanding. The terms proportional to $\Delta B_{0}^{\tilde{\tau}_{2} \tilde{\tau}_{1}}$ come from the graphs of Fig. 3. There is another set of four graphs corresponding to $\tilde{\tau}_{2}$. These are found after making the replacements $\tilde{\tau}_{1} \rightarrow \tilde{\tau}_{2}$, $s_{\tilde{\tau}} \rightarrow c_{\tilde{\tau}}$, and $c_{\tilde{\tau}} \rightarrow-s_{\tilde{\tau}}$. The diagrams in the first row are the ones that are equivalent to those in the bottom-sbottom loop. They have as characteristic feature the presence of two Rp-violating insertions (open circles) in the external legs. However, in contrast to the quark sector, R-parity violation can also appear in the charged internal lines running in the loops, since it occurs in the charged fermion sector. This explains the origin of the second row in Fig. 3. The presence of R-parity violating insertions in the internal lines of the second row in Fig. 3 correspond to the second topology in Fig. 1] The full 
diagrammatic explanation of the rest of the terms appearing in Eq. (30) is given in detail in Appendix C.

\section{Analytical versus Numerical results}

In this section we will check the accuracy of the approximation formulas given in Sec. 2.5 and 2.6. We do this by comparing the results obtained with their use with a full numerical calculation of the one-loop contributions to the neutrino mass, whose details can be found in Ref. [28].

As will be explained in more detail below, the relative importance of the various loops depends on the - currently unknown - supersymmetric parameters. In order to reduce the number of free parameters in the following we will adopt the minimal constrained supergravity (mSUGRA) version of the MSSM. As a rule of thumb it can be said that the bottom-sbottom loop usually gives the main contribution to the neutrino mass matrix when the neutralino is the LSP. On the other hand, if the scalar tau is the LSP, both bottom-sbottom and charged scalar loops are of approximately comparable magnitudes.

We have therefore constructed two different random scans over SUSY parameter space. Both sets start with the following rather generous parameter ranges: $M_{2}$ from $[0,1.2] \mathrm{TeV}$, $|\mu|$ from $[0,2.5] \mathrm{TeV}, m_{0}$ in the range $[0,1.0] \mathrm{TeV}, A_{0} / m_{0}$ and $B_{0} / m_{0}[-3,3]$ and $\tan \beta$ $[2.5,10]$. All randomly generated points were subsequently tested for consistency with the minimization (tadpole) conditions of the Higgs potential, as well as for phenomenological constraints from supersymmetric particle searches. We then selected points in which a) the lightest neutralino is the LSP (called set "Ntrl" in the following) or b) at least one of the charged sleptons was the LSP (called set "Stau" in the following). Note that in the Stau set $m_{0}<<M_{2}$ and large $\mu$ values are strongly preferred.

R-parity violating parameters are chosen in such a way that neutrino oscillation data are reproduced approximately. As discussed in the introduction, atmospheric neutrino experiments require a near-to-maximal atmospheric mixing angle $\theta_{\mathrm{ATM}}$, with $\Delta m_{\mathrm{ATM}}^{2}$ in the range given in Eq. (15). On the other hand reactor data constrain the electron-neutrino component in the third mass eigenstate to be small. And, finally, in combination with 
solar neutrino data, the KamLAND data require a $\theta_{\mathrm{SOL}}$ in the range given in Eq. (2) with $\Delta m_{\mathrm{SOL}}^{2}$ as given in Eq. (3). The latter ranges belong to the LMA-MSW region indicated by a solar-only global analysis of neutrino data given in Ref. [2]. For completeness we also include the (pre-KamLAND) LOW and VAC-type solutions of the solar neutrino anomaly. In the following we will first discuss the bottom-sbottom and the charged scalar loops separately, before considering a calculation taking into account both loops in comparison to the full calculation.

\subsection{Bottom-sbottom loop}

In Fig. 4 we show the ratio of the approximate-over-exact solar neutrino mass parameter $m_{\nu_{2}}^{\text {Appr }} / m_{\nu_{2}}^{\text {exact }}$ versus $\Delta m_{\mathrm{SOL}}^{2}$ for the case in which only the bottom-sbottom loop is taken into account, both in the approximate and in the exact calculation. The horizontal bands indicate attainable neutrino mass values when the parameters are scanned as indicated previously. As can be seen from the figure the approximate formula works quite well for points in both Ntrl and Stau sets, as long as the neutrino masses fall in the LMAMSW range indicated by the right vertical bands. Note that the LMA-MSW and LOW bands indicated in the figure correspond to the full analysis of solar data only, presented in Ref. 2]. The recent KamLAND reactor neutrino data rule out the LOW solution and restricts the LMA-MSW to somewhat narrower ranges indicated in Eq. (33). One finds that the mass values inferred from our present analytical approximation are always within $10 \%$ or less of the exact numerical calculation of the bottom-sbottom loop. Larger deviations show up only in the Ntrl set, for very small neutrino masses, which we trace to the neglection of the 1-loop neutrino/neutralino mixing terms in our approximate treatment. Although not strictly ruled out by a solar-only global neutrino data analysis [2], these LOW and VAC-type solutions are now strongly disfavored by the latest KamLAND reactor neutrino data. 

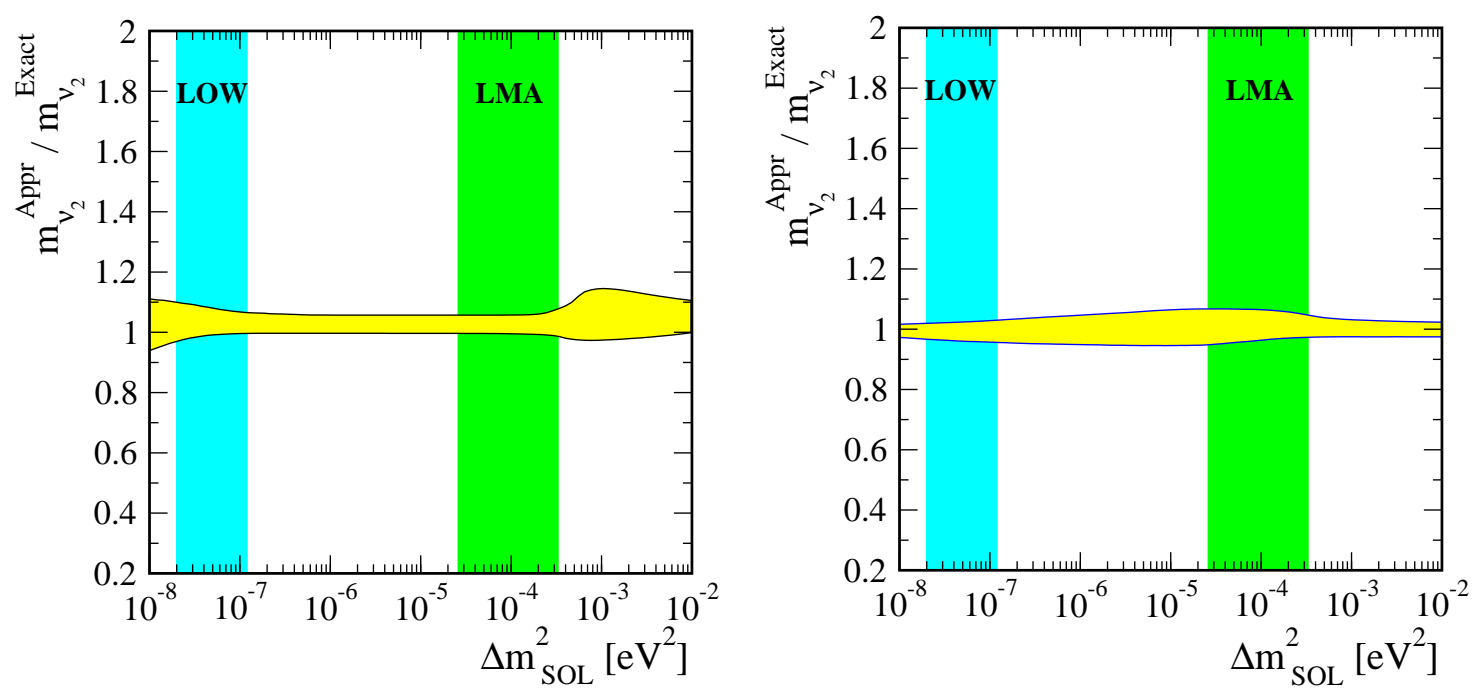

Figure 4: Ratio $m_{\nu_{2}}^{\text {Appr }} / m_{\nu_{2}}^{\text {exact }}$ versus $\Delta m_{\mathrm{SoL}}^{2}$ in $e V^{2}$ for the sets Ntrl (left) and Stau (right), for a calculation involving only the bottom-sbottom loop. The vertical grid lines indicate the $90 \%$ c.l. regions for the LOW and LMA solutions to the solar neutrino problem.
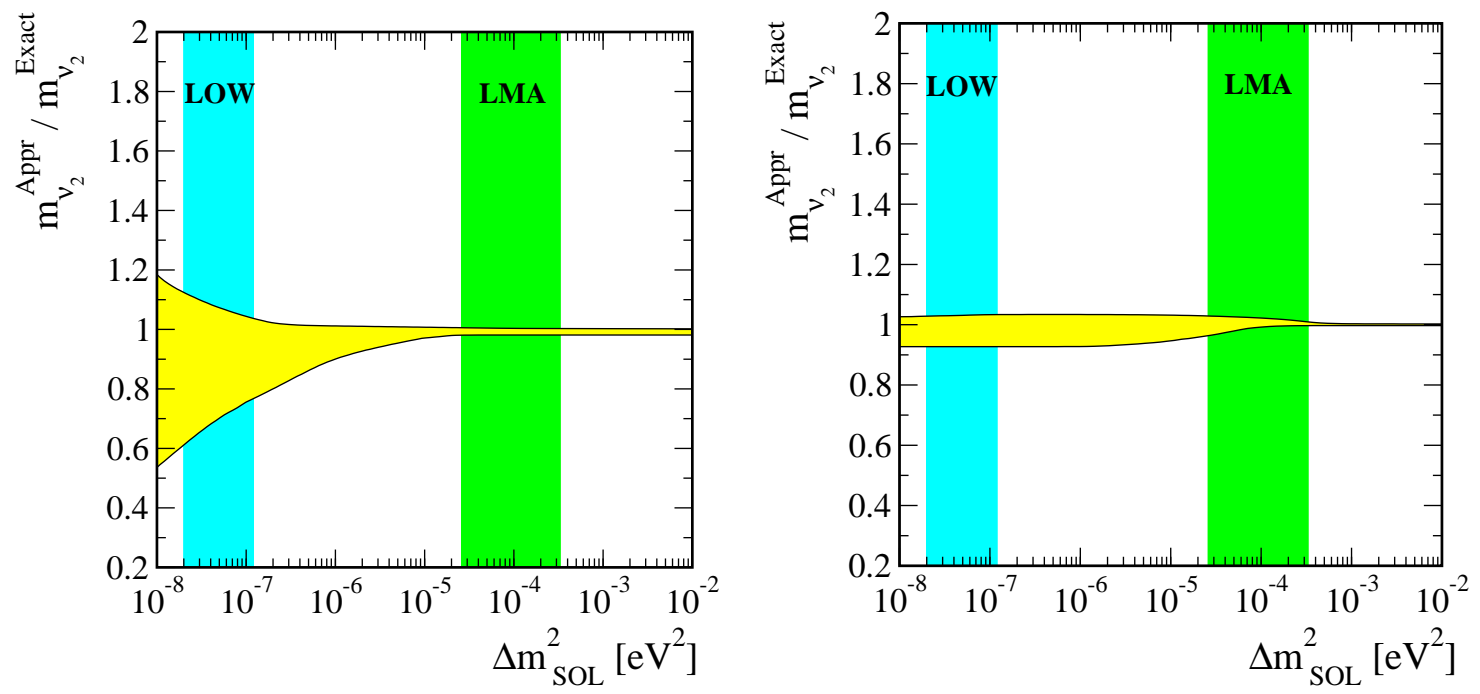

Figure 5: $\left(m_{\nu_{2}}^{A p p r} / m_{\nu_{2}}^{\text {exact }}\right)$ versus $\Delta m_{\mathrm{SOL}}^{2}\left[e V^{2}\right]$ for the sets Ntrl (left) and Stau (right), for a calculation involving only the charged scalar loop. 

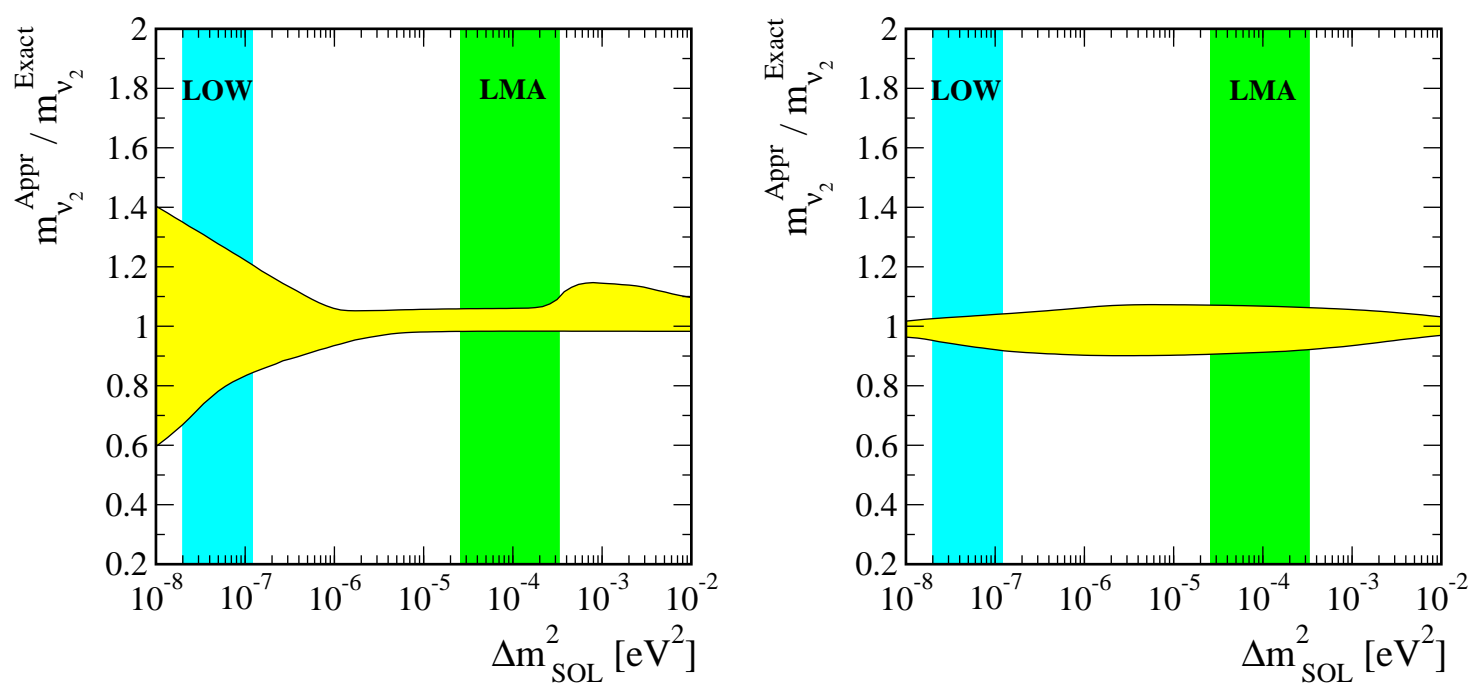

Figure 6: $\left(m_{\nu_{2}}^{\text {Appr }} / m_{\nu_{2}}^{\text {exact }}\right)$ versus $\Delta m_{\mathrm{SOL}}^{2}\left[e V^{2}\right]$ for the set Ntrl (left) and the set Stau (right). $m_{\nu_{2}}^{\text {Appr }}$ is the sum of the bottom-sbottom and charged scalar loops, while $m_{\nu_{2}}^{\text {exact }}$ is the numerical result for all loops. In case of LMA the approximation works always better than $10 \%$. For the LOW solution the typical error is of the order of $10 \%$, while in extreme cases errors up to $25 \%$ can be found.

\subsection{Charged scalar loop}

In Fig. 5 we show the ratio of the approximate-over-exact solar neutrino mass parameter $m_{\nu_{2}}^{A p p r} / m_{\nu_{2}}^{\text {exact }}$ plotted versus $\Delta m_{\mathrm{SOL}}^{2}$, for a calculation which takes into account only the charged scalar loop in both the approximate and the exact calculation. As can be seen from the figure the approximate formula is accurate for all points in the LMA-MSW region, indicated by the right vertical bands [2], both for the Stau and for the Ntrl sets. The only case where our analytic results gives a poorer approximation (to better than a factor-of-2) of the full numerical result is for the Ntrl set, when the neutrino mass falls in the LOW or VAC ranges, now strongly disfavored by the KamLAND results. We have checked numerically that for these very small neutrino masses all terms in eq. (30) are of approximately equal importance and there are significant cancellations among terms, which leads to a less reliable final result. 


\subsection{Comparison with full calculation}

In supersymmetric models with mSUGRA-like boundary conditions the bottom-sbottom and the charged scalar loop usually give the most important contribution to the neutrino mass matrix. This is demonstrated in Fig. [6 (left) for the set Ntrl and in Fig. [6 (right) for the set Stau. In both figures we show the ratio of the approximate-over-exact solar neutrino mass parameter $m_{\nu_{2}}^{\text {Appr }} / m_{\nu_{2}}^{\text {exact }}$ versus $\Delta m_{\mathrm{SOL}}^{2}$ in $\mathrm{eV}^{2}$, where $m_{\nu_{2}}^{\text {Appr }}$ is the approximate loop calculation involving the bottom-sbottom and the charged scalar loop, while $m_{\nu_{2}}^{\text {exact }}$ is the exact numerical computation taking into account all loops.

In the region of $\Delta m_{\mathrm{SOL}}^{2}$ appropriate for the currently preferred LMA-MSW solution to the solar neutrino problem one finds that the approximate calculation reproduces the exact result better than $10 \%$. Only in the set Ntrl one finds larger deviations, up to $25 \%$ in extreme cases, when $\Delta m_{\mathrm{SOL}}^{2}$ lies in the LOW region, strongly disfavored by KamLAND. This is due to the larger errors in the bottom-sbottom calculation in this set for small neutrino masses as discussed above.

\section{Simplified approximation formulas}

\subsection{The solar mass}

First we note that for nearly all points in our random sets we find that $m_{\nu_{2}} \ll m_{\nu_{3}}$. In other words, bilinear R-parity breaking favors a hierarchical neutrino spectrum. Moreover, we have found numerically that the terms proportional to $\tilde{\epsilon}_{i} \times \tilde{\epsilon}_{j}$ in the self energies in Eq. (27) give the most important contribution to $m_{\nu_{2}}$ in the bottom-sbottom loop calculation in most points of our sets. If these terms are dominant one can find a very simple approximation for the bottom-sbottom loop contribution to $m_{\nu_{2}}$. It is given by

$$
m_{\nu_{2}} \simeq \frac{3}{16 \pi^{2}} \sin \left(2 \theta_{\tilde{b}}\right) m_{b} \Delta B_{0}^{\tilde{\tau}_{2} \tilde{\tau}_{1}} \frac{\left(\tilde{\epsilon}_{1}^{2}+\tilde{\epsilon}_{2}^{2}\right)}{\mu^{2}}
$$

We have checked numerically that Eq. (33) reproduces the result of the full approximative formula to high accuracy if $m_{\nu_{2}} \leq 0.3 m_{\nu_{3}}$. Note also that Eq. (33) holds only if the 1-loop contributions to the neutrino mass matrix are smaller than the tree-level one. This condition requires that $|\vec{\epsilon}|^{2} /|\Lambda| \leq 1$ approximately, i.e. the bilinear parameters $\epsilon_{i}$ 
must be suppressed with respect to $\mu$. Note that such a suppression could, in principle, be motivated by suitable flavour symmetries [33].

Due to the more complicated structure of the charged scalar loop it is not possible to give a simple equation for $m_{\nu_{2}}$ similar to Eq. (33) for the bottom-sbottom loop. However, for $m_{\nu_{2}}$ larger than (few) $\times 10^{-4}$ we have found that the most important contributions to the charged scalar loop are the terms proportional to $\Delta B_{0}^{\tilde{\tau}_{2} \tilde{\tau}_{1}}, \Delta B_{0}^{H^{ \pm} \tilde{\tau}_{1}}$ and $\Delta B_{0}^{H^{ \pm} \tilde{\tau}_{2}}$ in Eq. (30). We note in passing that Eq. (33), with appropriate replacements, allows us to estimate the typical contributions to the charged scalar loop within a factor of $\sim 3$. However, such an estimate will be biased toward too small (large) $m_{\nu_{2}}$ for scalar tau (neutralino) LSPs.
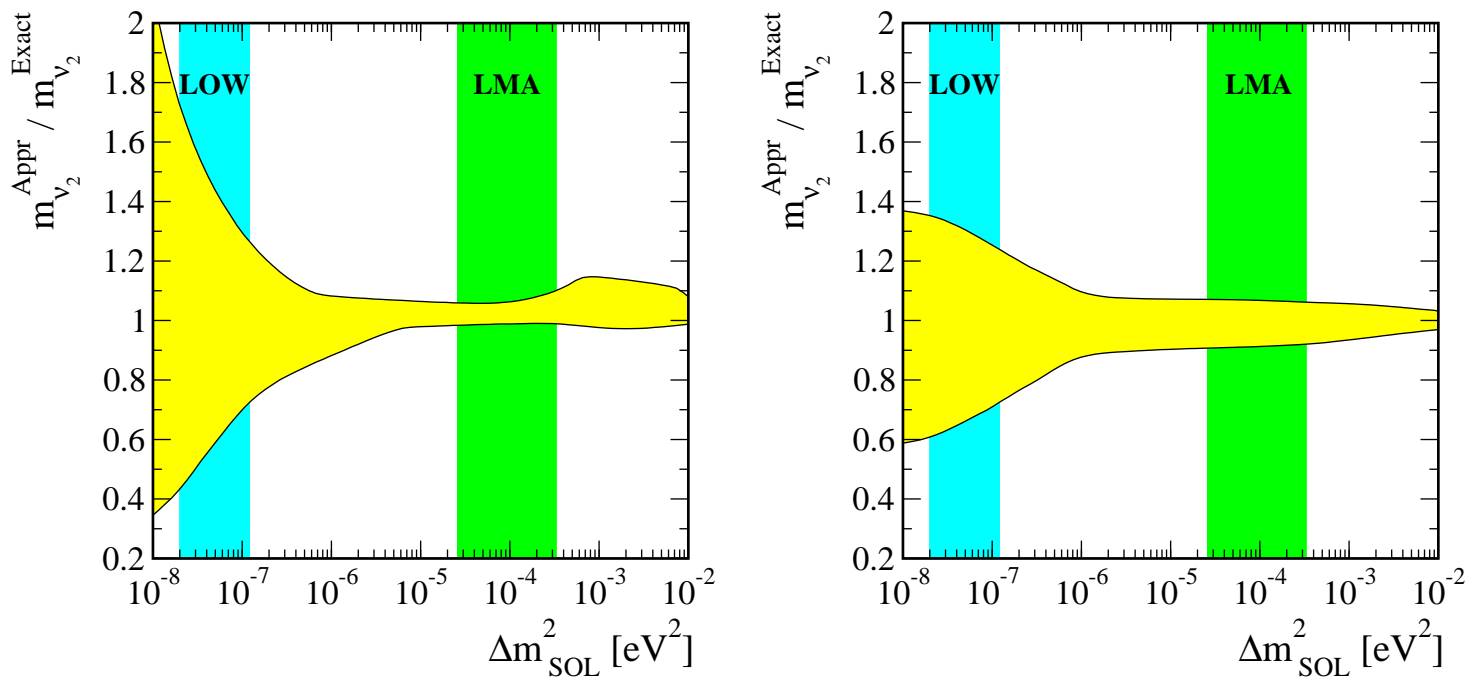

Figure 7: $\left(m_{\nu_{2}}^{A p p r} / m_{\nu_{2}}^{\text {exact}}\right)$ versus $\Delta m_{\mathrm{SOL}}^{2}\left[e V^{2}\right]$ for the set Ntrl (left) and Stau (right). Shown is the result of the simplified approximation formula in Eq. (33) for the sbottom-bottom loop and taking into account only coefficients $C_{H^{ \pm} \tilde{\tau}_{2}}, C_{H^{ \pm} \tilde{\tau}_{1}}$ and $C_{\tilde{\tau}_{2} \tilde{\tau}_{1}}$ in the charged scalar loop.

In Fig. (7) we show a comparison of our simplified approximation formula, including the simple form of the sbottom-bottom loop and the three most important coefficients for the charged scalar loop, as discussed above, to the full numerical calculation including all loops. As one can see, even the simplified version of our formula works surprisingly well in the LMA-MSW regime, although the agreement with the full calculation is now less good for the LOW region, as could have been expected from the results discussed previously. 


\subsection{The solar mixing angle}

In the basis where the tree-level neutrino mass matrix is diagonal the mass matrix at one-loop level can be written as

$$
\widetilde{m}_{\nu}=V_{\nu}^{(0) T} m_{\nu} V_{\nu}^{(0)}=\left(\begin{array}{ccc}
c_{1} \widetilde{\epsilon}_{1} \widetilde{\epsilon}_{1} & c_{1} \widetilde{\epsilon}_{1} \widetilde{\epsilon}_{2} & c_{1} \widetilde{\epsilon}_{1} \widetilde{\epsilon}_{3} \\
c_{1} \widetilde{\epsilon}_{2} \widetilde{\epsilon}_{1} & c_{1} \widetilde{\epsilon}_{2} \widetilde{\epsilon}_{2} & c_{1} \widetilde{\epsilon}_{2} \widetilde{\epsilon}_{3} \\
c_{1} \widetilde{\epsilon}_{3} \widetilde{\epsilon}_{1} & c_{1} \widetilde{\epsilon}_{3} \widetilde{\epsilon}_{2} & c_{0}|\vec{\Lambda}|^{2}+c_{1} \widetilde{\epsilon}_{3} \widetilde{\epsilon}_{3}
\end{array}\right)+\cdots
$$

where the $\widetilde{\epsilon}_{i}$ were defined before in Eq. (16). Coefficients $c_{0}$ and $c_{1}$ contain couplings and supersymmetric masses. Since they cancel in the final expression for the angle their exact definition is not necessary in the following. Dots stand for other terms which we will assume to be less important in the following, see the discussion at the end of this subsection. This matrix can be diagonalized approximately under the condition

$$
x \equiv \frac{c_{1}|\overrightarrow{\tilde{\epsilon}}|^{2}}{c_{0}|\vec{\Lambda}|^{2}} \ll 1
$$

i.e. if the 1-loop contribution to the neutrino mass matrix is smaller than the tree-level contribution, as also discussed above for Eq. (33). Then

$$
\widetilde{m}_{\nu}=c_{0}|\vec{\Lambda}|^{2}\left(\begin{array}{ccc}
x \frac{\tilde{\epsilon}_{1} \widetilde{\epsilon}_{1}}{|\vec{\epsilon}|^{2}} & x \frac{\widetilde{\epsilon}_{1} \widetilde{\epsilon}_{2}}{|\vec{\epsilon}|^{2}} & x \frac{\widetilde{\epsilon}_{1} \widetilde{\epsilon}_{3}}{|\vec{\epsilon}|^{2}} \\
x \frac{\widetilde{\epsilon}_{2} \widetilde{\epsilon}_{1}}{\mid \overrightarrow{\left.\widetilde{\epsilon}\right|^{2}}} & x \frac{\widetilde{\epsilon}_{2} \widetilde{\epsilon}_{2}}{|\vec{\epsilon}|^{2}} & x \frac{\widetilde{\epsilon}_{2} \widetilde{\epsilon}_{3}}{|\vec{\epsilon}|^{2}} \\
x \frac{\widetilde{\epsilon}_{3} \widetilde{\epsilon}_{1}}{|\vec{\epsilon}|^{2}} & x \frac{\widetilde{\epsilon}_{3} \widetilde{\epsilon}_{2}}{|\vec{\epsilon}|^{2}} & 1+x \frac{\widetilde{\epsilon}_{3} \widetilde{\epsilon}_{3}}{|\vec{\epsilon}|^{2}}
\end{array}\right)
$$

We now calculate the eigenvalues and eigenvectors of this matrix as series expansions in the small $x$ parameter. For the eigenvalues we get

$$
\begin{aligned}
& m_{1}=0 \\
& m_{2}=x c_{0} \frac{|\vec{\Lambda}|^{2}}{|\vec{\epsilon}|^{2}}+\mathcal{O}\left(x^{2}\right)=c_{1}\left(\widetilde{\epsilon}_{1}^{2}+\widetilde{\epsilon}_{2}^{2}\right)+\mathcal{O}\left(x^{2}\right) \\
& m_{3}=c_{0}|\vec{\Lambda}|^{2}+c_{1} \widetilde{\epsilon}_{3}^{2}+\mathcal{O}\left(x^{2}\right)
\end{aligned}
$$

and for the first two eigenvalues (the third can also be easily obtained but it will not be necessary for the discussion of the solar mixing angle),

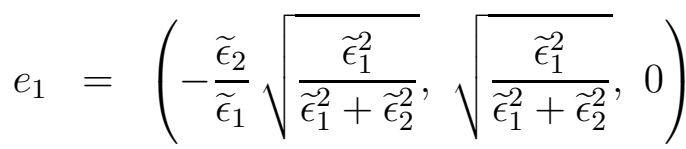

$$
\begin{aligned}
& e_{2}=\left(e_{2,1}, e_{2,2}, e_{2,3}\right)
\end{aligned}
$$


where up to $\mathcal{O}\left(x^{2}\right)$ we have,

$$
\begin{aligned}
& e_{2,1}=-\frac{\widetilde{\epsilon}_{1} \widetilde{\epsilon}_{3}}{\sqrt{\widetilde{\epsilon}_{3}^{2}\left(\widetilde{\epsilon}_{1}^{2}+\widetilde{\epsilon}_{2}^{2}\right)}}+\frac{1}{2} \frac{\widetilde{\epsilon}_{1} \widetilde{\epsilon}_{3} \sqrt{\widetilde{\epsilon}_{3}^{2}\left(\widetilde{\epsilon}_{1}^{2}+\widetilde{\epsilon}_{2}^{2}\right)}}{\mid \vec{\epsilon}^{4}} x^{2}+\mathcal{O}\left(x^{3}\right) \\
& e_{2,2}=-\frac{\widetilde{\epsilon}_{2} \widetilde{\epsilon}_{3}}{\sqrt{\widetilde{\epsilon}_{3}^{2}\left(\widetilde{\epsilon}_{1}^{2}+\widetilde{\epsilon}_{2}^{2}\right)}}+\frac{1}{2} \frac{\widetilde{\epsilon}_{2} \widetilde{\epsilon}_{3} \sqrt{\widetilde{\epsilon}_{3}^{2}\left(\widetilde{\epsilon}_{1}^{2}+\widetilde{\epsilon}_{2}^{2}\right)}}{\mid \vec{\epsilon}^{4}} x^{2}+\mathcal{O}\left(x^{3}\right) \\
& e_{2,3}=\frac{\sqrt{\widetilde{\epsilon}_{3}^{2}\left(\widetilde{\epsilon}_{1}^{2}+\widetilde{\epsilon}_{2}^{2}\right)}}{|\vec{\epsilon}|^{2}} x+\frac{\left(\widetilde{\epsilon}_{1}^{2}+\widetilde{\epsilon}_{2}^{2}-\widetilde{\epsilon}_{3}^{2}\right) \sqrt{\widetilde{\epsilon}_{3}^{2}\left(\widetilde{\epsilon}_{1}^{2}+\widetilde{\epsilon}_{2}^{2}\right)}}{\mid \widetilde{\epsilon}^{4}} x^{2}+\mathcal{O}\left(x^{3}\right)
\end{aligned}
$$

Knowing the eigenvectors we can write down the rotation matrix that diagonalizes $\widetilde{m}_{\nu}$,

$$
\widetilde{V}_{\nu}^{T} \widetilde{m}_{\nu} \widetilde{V}_{\nu}=\operatorname{diag}\left(m_{1}, m_{2}, m_{3}\right)
$$

where

$$
\widetilde{V}_{\nu}^{T}=\left(\begin{array}{ccc}
e_{1,1} & e_{1,2} & e_{1,3} \\
e_{2,1} & e_{2,2} & e_{2,3} \\
e_{3,1} & e_{3,2} & e_{3,3}
\end{array}\right)
$$

The neutrino mixing matrix is then given by

$$
U=\left(V_{\nu}^{T} \widetilde{V}_{\nu}^{T}\right)^{T}
$$

Using the fact that $U_{e 3}$ has to be small one can get the following expression for the solar mixing angle:

$$
\tan ^{2} \theta_{\mathrm{SOL}}=\frac{U_{e 2}^{2}}{U_{e 1}^{2}}
$$

Now using the Eqs. (41), (39) and substituting in Eq. (42) we obtain the very simple expression for the solar mixing angle,

$$
\tan ^{2} \theta_{\mathrm{SOL}}=\frac{\widetilde{\epsilon}_{1}^{2}}{\widetilde{\epsilon}_{2}^{2}}
$$

This formula is a very good approximation if the one-loop matrix has the structure $\epsilon_{i} \times \epsilon_{j}$, as is the case of the bottom-sbottom loop (and, to a lesser extend also for the charged scalar loop, which has one coefficient with the same index structure), and if $m_{\nu_{3}} \gg m_{\nu_{2}}$. This is illustrated in Fig. 8 . 
In the left panel we show a calculation comparing for all points in the set Ntrl the approximate to the exact solar angle, while the right panel shows a subset of points using the cut $\sin \left(2 \theta_{\tilde{b}}\right) \Delta B_{0}^{\tilde{\tau}_{2} \tilde{\tau}_{1}}>0.02$. Note that this cut is designed such as to prefer points in which there is a sizeable contribution to the full 1-loop neutrino mass due to the bottomsbottom loop. For points in which the charged scalar loop dominates eq. (44) gives only a factor-of-two estimate of the true solar angle.

Note finally that eq. (44) will fail completely, if $\Lambda_{\mu} \equiv \Lambda_{\tau}$ and $\epsilon_{\mu} \equiv \epsilon_{\tau}$, since then $\tilde{\epsilon}_{2}^{2}=0$, see Eq. (16). This is the origin of the "sign condition" discussed in [28].
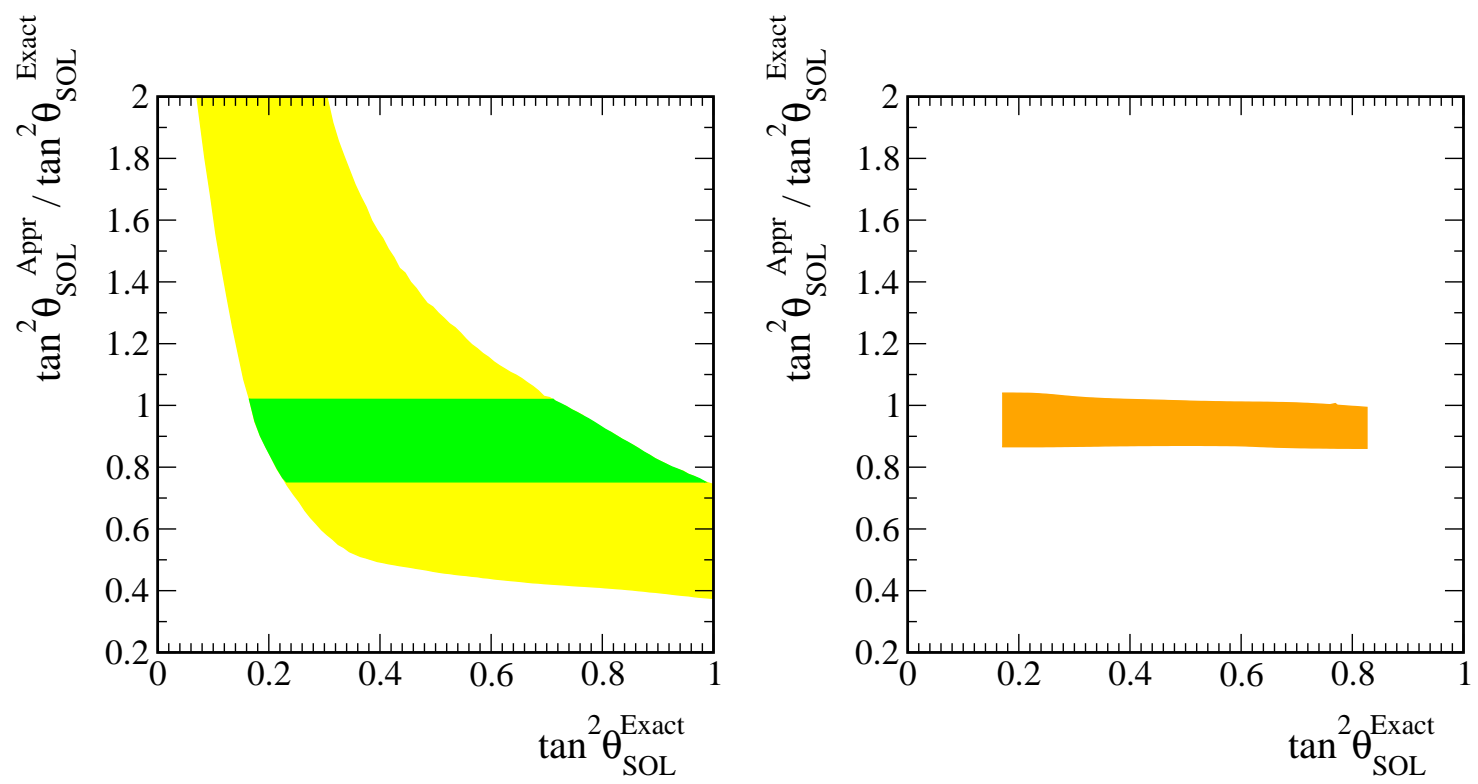

Figure 8: $\left(\tan ^{2} \theta_{\mathrm{SOL}}{ }^{A p p r} / \tan ^{2} \theta_{\mathrm{SOL}}{ }^{\text {exact }}\right)$ versus $\tan ^{2} \theta_{\mathrm{SOL}}{ }^{\text {exact }}$. On the left panel the darker region contains over $90 \%$ of the points in our sample. In the right panel the points in the region shown satisfy the cut $\sin \left(2 \theta_{\tilde{b}}\right) \Delta B_{0}^{\tilde{\tau}_{2}} \tilde{\tau}_{1}>0.02$.

\section{$5 \quad$ Discussion and conclusions}

We have presented an approximate calculation of the neutrino mass matrix at one-loop in supersymmetry with bilinearly broken R-parity. The method is based on a systematic perturbative expansion of R-parity violating vertices to leading order. We have identified the bottom-sbottom and the charged scalar loop as the most important ones, at least in supersymmetric models with mSUGRA-like boundary conditions. Taking into account 
only these loops, we have given explicit formulas and discussed their validity as well as the accuracy with which they describe solar neutrino mass and mixing parameters. This was done by comparing our analytical results to the exact numerical calculation. We have found that for the case of the large mixing MSW solution our formulas - even within the simplified form Eq. (33) and Eq. (44) - yield good agreement with the full numerical calculation, but are much simpler to implement than the full numerical one-loop calculation. The only solar neutrino "solutions" for which our analytical approximation is less accurate are those that are now ruled out by the recent reactor neutrino data from KamLAND.

Let us finally discuss some possible caveats to the success of our approximate treatment. One is the assumption that supersymmetry breaking mass terms are flavour diagonal, which we have adopted, motivated by constraints from flavour changing processes. Although such terms could be included into our approximate treatment, we have not done so, mainly due to the fact that the resulting formulas would be much more complicated and, therefore, of very limited practical use. A second concern is that our sample points were all generated using mSugra assumptions for the soft breaking masses. Clearly there are other possibilities to break supersymmetry and even though we expect that the bottom-sbottom loop and the charged scalar loop will still be well described by our approximation formulas, other loops, which we didn't take into account, might be more important than what we have found in our data sets.

\section{Acknowledgements}

This work was supported by Spanish grant BFM2002-00345, by the European Commission RTN grant HPRN-CT-2000-00148 and the ESF Neutrino Astrophysics Network. M. H. is supported by a Spanish MCyT Ramon y Cajal contract. W. P. is supported by the 'Erwin Schrödinger fellowship No. J2095' of the 'Fonds zur Förderung der wissenschaftlichen Forschung' of Austria FWF and partly by the Swiss 'Nationalfonds'. M.A.D. was supported by Conicyt grant No. 1010974. J.R. was partly supported by the fellowship SFRH/BSAB/269/2002 from FCT (Portugal) with funds from the EU. 


\section{A Rotation Matrices}

In the basis $\left(H_{d}^{+}, H_{u}^{+}, \widetilde{e}_{L}^{+}, \widetilde{\mu}_{L}^{+}, \widetilde{\tau}_{L}^{+}, \widetilde{e}_{R}^{+}, \widetilde{\mu}_{R}^{+}, \widetilde{\tau}_{R}^{+}\right)$, one can write, to first order in R-parity violating parameters, the Goldstone rotation matrix as

$$
\mathbf{R}_{G}=\left[\begin{array}{cccccccc}
c_{\beta} & -s_{\beta} & v_{1} / v & v_{2} / v & v_{3} / v & 0 & 0 & 0 \\
s_{\beta} & c_{\beta} & 0 & 0 & 0 & 0 & 0 & 0 \\
-c_{\beta} v_{1} / v & s_{\beta} v_{1} / v & 1 & 0 & 0 & 0 & 0 & 0 \\
-c_{\beta} v_{2} / v & s_{\beta} v_{2} / v & 0 & 1 & 0 & 0 & 0 & 0 \\
-c_{\beta} v_{3} / v & s_{\beta} v_{3} / v & 0 & 0 & 1 & 0 & 0 & 0 \\
0 & 0 & 0 & 0 & 0 & 1 & 0 & 0 \\
0 & 0 & 0 & 0 & 0 & 0 & 1 & 0 \\
0 & 0 & 0 & 0 & 0 & 0 & 0 & 1
\end{array}\right]
$$

where $v^{2}=v_{d}^{2}+v_{u}^{2}$ to this order, and $\tan \beta=v_{u} / v_{d}$, as usual. We have also used the shorthand notation $c_{\beta}\left(s_{\beta}\right)=\cos \beta(\sin \beta)$.

Neglecting the electron and muon Yukawa couplings, the rotation that diagonalizes the sleptons at tree level is given by (in the same basis as above)

$$
\mathbf{R}_{\tilde{\tau}}=\left[\begin{array}{cccccccc}
1 & 0 & 0 & 0 & 0 & 0 & 0 & 0 \\
0 & 1 & 0 & 0 & 0 & 0 & 0 & 0 \\
0 & 0 & 1 & 0 & 0 & 0 & 0 & 0 \\
0 & 0 & 0 & 1 & 0 & 0 & 0 & 0 \\
0 & 0 & 0 & 0 & c_{\tilde{\tau}} & 0 & 0 & s_{\tilde{\tau}} \\
0 & 0 & 0 & 0 & 0 & 1 & 0 & 0 \\
0 & 0 & 0 & 0 & 0 & 0 & 1 & 0 \\
0 & 0 & 0 & 0 & -s_{\tilde{\tau}} & 0 & 0 & c_{\tilde{\tau}}
\end{array}\right]
$$

After the rotations $\mathbf{R}_{\tilde{\tau}} \mathbf{R}_{G}$ are performed, the charged scalar mass matrix is diagonalized up to small R-parity violating entries. In the approximation where there is no intergenerational mixing and $h_{\mu} \approx h_{e} \approx 0$, these are 


$$
\Delta \mathbf{M}_{\mathbf{S}^{ \pm} \tilde{\tau}}^{2}=\left[\begin{array}{cccccccc}
0 & 0 & 0 & 0 & 0 & 0 & 0 & 0 \\
0 & 0 & \widetilde{X}_{H L_{1}} & \widetilde{X}_{H L_{2}} & \widetilde{X}_{H L_{3}} & 0 & 0 & \widetilde{X}_{H R_{3}} \\
0 & \widetilde{X}_{H L_{1}} & 0 & 0 & 0 & 0 & 0 & 0 \\
0 & \widetilde{X}_{H L_{2}} & 0 & 0 & 0 & 0 & 0 & 0 \\
0 & \widetilde{X}_{H L_{3}} & 0 & 0 & 0 & 0 & 0 & 0 \\
0 & 0 & 0 & 0 & 0 & 0 & 0 & 0 \\
0 & 0 & 0 & 0 & 0 & 0 & 0 & 0 \\
0 & \widetilde{X}_{H R_{3}} & 0 & 0 & 0 & 0 & 0 & 0
\end{array}\right]
$$

where

$$
\begin{array}{ll}
\widetilde{X}_{H L_{i}}=X_{H L_{i}} & \widetilde{X}_{H R_{i}}=X_{H R_{i}},(i=1,2) \\
\widetilde{X}_{H L_{3}}=c_{\tilde{\tau}} X_{u L_{3}}+s_{\tilde{\tau}} X_{d R_{3}} & \widetilde{X}_{H R_{3}}=-s_{\tilde{\tau}} X_{H L_{3}}+c_{\tilde{\tau}} X_{H R_{3}}
\end{array}
$$

with

$$
X_{H L_{i}}=s_{\beta} X_{u L_{i}}+c_{\beta} X_{d L_{i}} \quad X_{H R_{3}}=s_{\beta} X_{u R_{3}}+c_{\beta} X_{d R_{3}}, \quad(\mathrm{i}=1,3)
$$

and

$$
\begin{array}{ll}
X_{u L_{i}}=\frac{1}{4} g^{2} v_{d} v_{i}-\mu \epsilon_{i}-\frac{1}{2} h_{\tau}^{2} v_{d} v_{i} \delta_{i 3}, & X_{d L_{i}}=\frac{v_{i}}{v_{d}} \frac{c_{\beta}}{s_{\beta}} m_{\tilde{\nu}}^{2}-\mu \epsilon_{i} \frac{c_{\beta}}{s_{\beta}}+\frac{1}{4} g^{2} v_{u} v_{i}, \\
X_{u R_{3}}=-\frac{1}{\sqrt{2}} h_{\tau}\left(A_{\tau} v_{3}+\epsilon_{3} v_{u}\right), & X_{d R_{3}}=-\frac{1}{\sqrt{2}} h_{\tau}\left(\mu v_{3}+\epsilon_{3} v_{d}\right) .
\end{array}
$$

These mixings are removed with the rotation matrix $\mathbf{R}_{\mathbf{X}}$ given by

$$
\mathbf{R}_{\mathbf{X}}=\left[\begin{array}{cccccccc}
1 & 0 & 0 & 0 & 0 & 0 & 0 & 0 \\
0 & 1 & \Theta_{H L_{1}} & \Theta_{H L_{2}} & \Theta_{H L_{3}} & 0 & 0 & \Theta_{H R_{3}} \\
0 & -\Theta_{H L_{1}} & 1 & 0 & 0 & 0 & 0 & 0 \\
0 & -\Theta_{H L_{2}} & 0 & 1 & 0 & 0 & 0 & 0 \\
0 & -\Theta_{H L_{3}} & 0 & 0 & 1 & 0 & 0 & 0 \\
0 & 0 & 0 & 0 & 0 & 1 & 0 & 0 \\
0 & 0 & 0 & 0 & 0 & 0 & 1 & 0 \\
0 & -\Theta_{H R_{3}} & 0 & 0 & 0 & 0 & 0 & 1
\end{array}\right]
$$

in the small mixing approximation $\sin \Theta \simeq \Theta$. Note that here we have defined

$$
\Theta_{H L_{i}} \equiv \frac{\widetilde{X}_{H L_{i}}}{m_{H^{ \pm}}^{2}-m_{\tilde{\ell}_{L i}}^{2}}, \quad \Theta_{H R_{i}} \equiv \frac{\widetilde{X}_{H R_{i}}}{m_{H^{ \pm}}^{2}-m_{\tilde{\ell}_{R i}}^{2}}
$$


Putting everything together we get the final form of the charged scalar diagonalization matrix $\mathbf{R}_{X} \mathbf{R}_{\tilde{\tau}} \mathbf{R}_{G}$ which can be expressed as

$$
\begin{aligned}
& \mathbf{R}_{X} \mathbf{R}_{\tilde{\tau}} \mathbf{R}_{G}= \\
& {\left[\begin{array}{cccccccc}
c_{\beta} & -s_{\beta} & v_{1} / v & v_{2} / v & v_{3} / v & 0 & 0 & 0 \\
s_{\beta} & c_{\beta} & \Theta_{H L_{1}} & \Theta_{H L_{2}} & \widetilde{\Theta}_{H L_{3}} & 0 & 0 & \widetilde{\Theta}_{H R_{3}} \\
-s_{\beta} \Theta_{H L_{1}}-c_{\beta} \frac{v_{1}}{v} & -c_{\beta} \Theta_{H L_{1}}+s_{\beta} \frac{v_{1}}{v} & 1 & 0 & 0 & 0 & 0 & 0 \\
-s_{\beta} \Theta_{H L_{2}}-c_{\beta} \frac{v_{2}}{v} & -c_{\beta} \Theta_{H L_{2}}+s_{\beta} \frac{v_{2}}{v} & 0 & 1 & 0 & 0 & 0 & 0 \\
-s_{\beta} \Theta_{H L_{3}}-c_{\tilde{\tau}} c_{\beta} \frac{v_{3}}{v} & -c_{\beta} \Theta_{H L_{3}}+c_{\tilde{\tau}} s_{\beta} \frac{v_{3}}{v} & 0 & 0 & c_{\tilde{\tau}} & 0 & 0 & s_{\tilde{\tau}} \\
0 & 0 & 0 & 0 & 0 & 1 & 0 & 0 \\
0 & 0 & 0 & 0 & 0 & 0 & 1 & 0 \\
-s_{\beta} \Theta_{H R_{3}}+s_{\tilde{\tau}} c_{\beta} \frac{v_{3}}{v} & -c_{\beta} \Theta_{H R_{3}}-s_{\tilde{\tau}} s_{\beta} \frac{v_{3}}{v} & 0 & 0 & -s_{\tilde{\tau}} & 0 & 0 & c_{\tilde{\tau}}
\end{array}\right]}
\end{aligned}
$$

where we have defined,

$$
\widetilde{\Theta}_{H L_{3}}=c_{\tilde{\tau}} \Theta_{H L_{3}}-s_{\tilde{\tau}} \Theta_{H R_{3}}, \widetilde{\Theta}_{H R_{3}}=s_{\tilde{\tau}} \Theta_{H L_{3}}+c_{\tilde{\tau}} \Theta_{H R_{3}} .
$$




\section{B Charged Higgs/slepton couplings}

The couplings of the five (generalized to include also the three charged leptons) charginos to the eight charged scalars (including Higgs bosons and sleptons of both chiralities) and seven neutralinos (generalized to include also the three neutrinos) are given by [28]

$$
\begin{aligned}
O_{\text {Lijk }}^{c n s}= & R_{k 1}^{S^{ \pm}} h_{\tau} \mathcal{N}_{j 7} \mathcal{V}_{i 5}-R_{k 2}^{S^{ \pm}}\left(\frac{g}{\sqrt{2}} \mathcal{N}_{j 2} \mathcal{V}_{i 2}+\frac{g^{\prime}}{\sqrt{2}} \mathcal{N}_{j 1} \mathcal{V}_{i 2}+g \mathcal{N}_{j 4} \mathcal{V}_{i 1}\right) \\
& -R_{k 5}^{S^{ \pm}} h_{\tau} \mathcal{N}_{j 3} \mathcal{V}_{i 5}-g^{\prime} \sqrt{2}\left(R_{k 6}^{S^{ \pm}} \mathcal{N}_{j 1} \mathcal{V}_{i 3}+R_{k 7}^{S^{ \pm}} \mathcal{N}_{j 1} \mathcal{V}_{i 4}+R_{k 8}^{S^{ \pm}} \mathcal{N}_{j 1} \mathcal{V}_{i 5}\right)
\end{aligned}
$$

where $i$ labels the charginos, $j$ labels neutralinos, and $k$ labels the charged scalars, respectively. For the the right-handed couplings the corresponding couplings are given by

$$
\begin{aligned}
O_{\text {Rijk }}^{c n s}= & R_{k 1}^{S^{ \pm}}\left(\frac{g}{\sqrt{2}} \mathcal{N}_{j 2} \mathcal{U}_{i 2}+\frac{g^{\prime}}{\sqrt{2}} \mathcal{N}_{j 1} \mathcal{U}_{i 2}-g \mathcal{N}_{j 3} \mathcal{U}_{i 1}\right) \\
& +R_{k 3}^{S^{ \pm}}\left(\frac{g}{\sqrt{2}} \mathcal{N}_{j 2} \mathcal{U}_{i 3}+\frac{g^{\prime}}{\sqrt{2}} \mathcal{N}_{j 1} \mathcal{U}_{i 3}-g \mathcal{N}_{j 5} \mathcal{U}_{i 1}\right) \\
& +R_{k 4}^{S^{ \pm}}\left(\frac{g}{\sqrt{2}} \mathcal{N}_{j 2} \mathcal{U}_{i 4}+\frac{g^{\prime}}{\sqrt{2}} \mathcal{N}_{j 1} \mathcal{U}_{i 4}-g \mathcal{N}_{j 6} \mathcal{U}_{i 1}\right) \\
& +R_{k 5}^{S^{ \pm}}\left(\frac{g}{\sqrt{2}} \mathcal{N}_{j 2} \mathcal{U}_{i 5}+\frac{g^{\prime}}{\sqrt{2}} \mathcal{N}_{j 1} \mathcal{U}_{i 5}-g \mathcal{N}_{j 7} \mathcal{U}_{i 1}\right) \\
& +R_{k 8}^{S^{ \pm}} h_{\tau}\left(\mathcal{N}_{j 7} \mathcal{U}_{i 2}-\mathcal{N}_{j 3} \mathcal{U}_{i 5}\right)
\end{aligned}
$$

After approximating the rotation matrices $\mathcal{U}$, and $\mathcal{V}$ in the chargino sector, and $\mathcal{N}$ in the neutralino sector we find the expressions given in Eqs. (57)-(60). Note that we have divided them into cases where the charged fermion is a lepton or a chargino. For the left couplings when the charged fermion is a chargino we have,

$$
O_{\text {Lijk }}^{c n s}=R_{k 2}^{S^{ \pm}}\left[\frac{g}{\sqrt{2}} a_{2} V_{i^{\prime} 2}+\frac{g^{\prime}}{\sqrt{2}} a_{1} V_{i^{\prime} 2}+g a_{4} V_{i^{\prime} 1}\right]|\vec{\Lambda}| \delta_{j 3}
$$

where $V$ is the reduced $2 \times 2$ chargino diagonalization matrix of the MSSM, and $i^{\prime}=1,2$. If the charged fermion is a lepton we have

$$
\begin{aligned}
O_{\text {Lijk }}^{c n s}= & R_{k 1}^{S^{ \pm}} h_{\tau} V_{\nu, j 3}^{T} \delta_{i 3}+R_{k 5}^{S^{ \pm}} h_{\tau}\left(b \tilde{\epsilon}_{j}+a_{3}|\vec{\Lambda}| \delta_{j 3}\right) \delta_{i 3} \\
& +\left[R_{k 6}^{S^{ \pm}} \delta_{i 1}+R_{k 7}^{S^{ \pm}} \delta_{i 2}+R_{k 8}^{S^{ \pm}} \delta_{i 3}\right] \sqrt{2} g^{\prime} a_{1}|\vec{\Lambda}| \delta_{j 3}
\end{aligned}
$$

For the right-handed couplings when the charged fermion is a chargino we get

$$
\begin{aligned}
O_{R i j k}^{c n s}= & R_{k 1}^{S^{ \pm}}\left[-\frac{1}{\sqrt{2}}\left(g a_{2}+g^{\prime} a_{1}\right)|\vec{\Lambda}| \delta_{j 3} U_{i^{\prime} 2}+g\left(b \tilde{\epsilon}_{j}+a_{3}|\vec{\Lambda}| \delta_{j 3}\right) U_{i^{\prime} 1}\right] \\
& -R_{k 3}^{S^{ \pm}} g V_{\nu, j 1}^{T} U_{i^{\prime} 1}-R_{k 4}^{S^{ \pm}} g V_{\nu, j 2}^{T} U_{i^{\prime} 1}-R_{k 5}^{S^{ \pm}} g V_{\nu, j 3}^{T} U_{i^{\prime} 1}+R_{k 8}^{S^{ \pm}} h_{\tau} V_{\nu, j 3}^{T} U_{i^{\prime} 2}
\end{aligned}
$$


where $U$ is the second $2 \times 2$ chargino rotation matrix of the MSSM. Finally, if the charged fermion is a lepton one has

$$
\begin{aligned}
O_{R i j k}^{c n s}= & -R_{k 3}^{S^{ \pm}}\left[\frac{1}{\sqrt{2}}\left(g a_{2}+g^{\prime} a_{1}\right)|\vec{\Lambda}| \delta_{j 3} \delta_{i 1}-g V_{\nu, j 1}^{T} a_{1}^{L} \Lambda_{i}\right] \\
& -R_{k 4}^{S^{ \pm}}\left[\frac{1}{\sqrt{2}}\left(g a_{2}+g^{\prime} a_{1}\right)|\vec{\Lambda}| \delta_{j 3} \delta_{i 2}-g V_{\nu, j 2}^{T} a_{1}^{L} \Lambda_{i}\right] \\
& -R_{k 5}^{S^{ \pm}}\left[\frac{1}{\sqrt{2}}\left(g a_{2}+g^{\prime} a_{1}\right)|\vec{\Lambda}| \delta_{j 3} \delta_{i 3}-g V_{\nu, j 3}^{T} a_{1}^{L} \Lambda_{i}\right] \\
& -R_{k 8}^{S^{ \pm}} h_{\tau}\left[V_{\nu, j 3}^{T}\left(a_{2}^{L} \Lambda_{i}+b \epsilon_{i}\right)-\left(b \tilde{\epsilon}_{j}+a_{3}|\vec{\Lambda}| \delta_{j 3}\right) \delta_{i 3}\right]
\end{aligned}
$$




\section{Charged Scalar-Charged Fermion Loops}

There are nine different terms contributing to the Charged Scalar-Charged Fermion loop, as it was shown in Eq. (30). All these terms give a finite contribution to the $2 \times 2$ submatrix corresponding to the light neutrinos. In this Appendix we will explain with graphs the origin of the different terms. The conventions used were explained in section 2.6.

\section{C.1 $\Delta B_{0}^{\tilde{\tau}_{2} \tilde{\tau}_{1}}$}

The terms proportional to $\Delta B_{0}^{\tilde{\tau}_{2} \tilde{\tau}_{1}}$ come from the graphs of Fig. 3 as explained in section 2.6.

\section{C.2 $\Delta B_{0}^{H^{+} \tilde{\tau}_{1}}$ and $\Delta B_{0}^{H^{+} \tilde{\tau}_{2}}$}

Now consider the terms proportional to $\Delta B_{0}^{H^{+} \tilde{\tau}_{1}}$ and $\Delta B_{0}^{H^{+} \tilde{\tau}_{2}}$ in Eq. (30). Of these terms, the ones which are related to the charged Higgs mixing with staus, can be understood as coming from the four graphs of Fig. 9. Associated to these charged Higgs graphs are those related to the $\tilde{\tau}_{1}$ mixing with charged Higgs. These are given in Fig. 10,
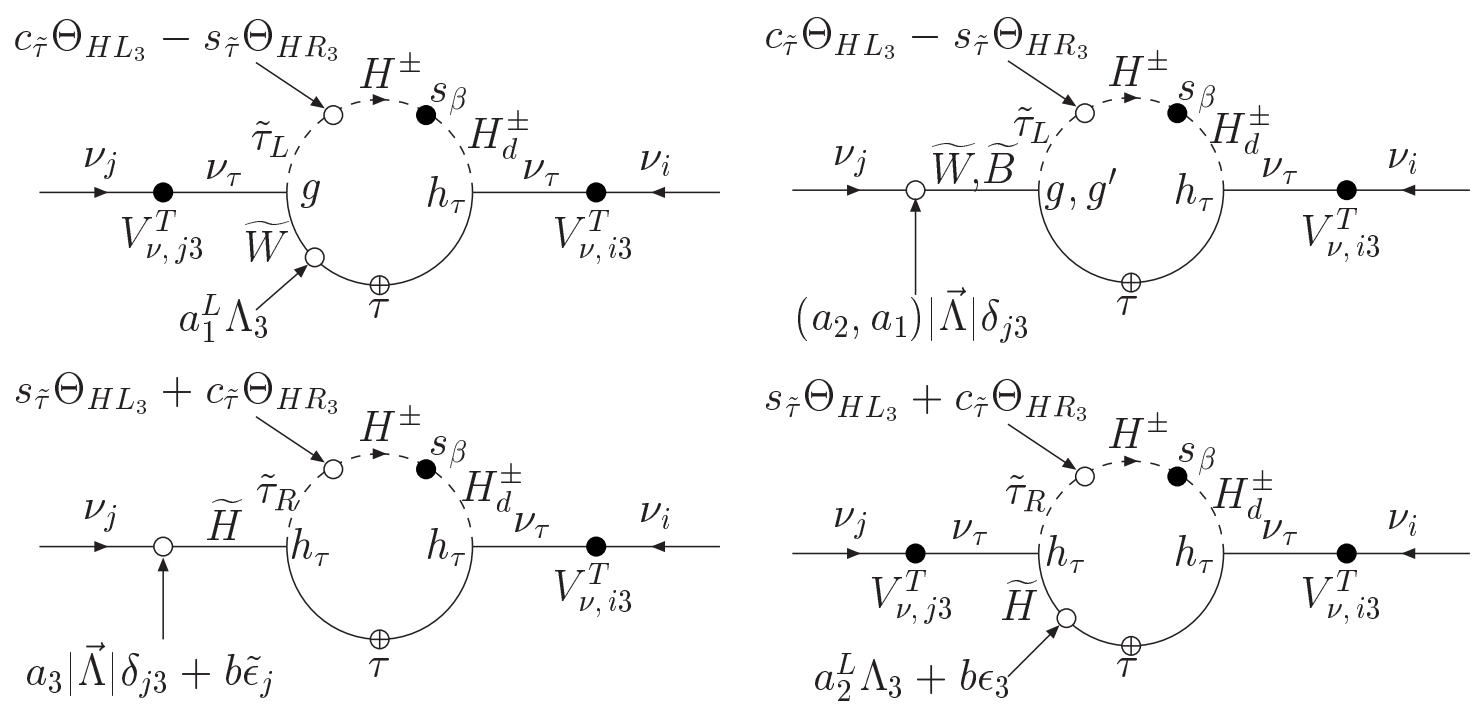

Figure 9: $H^{ \pm}$contribution to $\Delta B_{0}^{H^{ \pm} \tilde{\tau}_{1}}$

There is another set of four graphs corresponding to $\tilde{\tau}_{2}$ that are obtained from those in Fig. 10 by replacing $\tilde{\tau}_{1} \rightarrow \tilde{\tau}_{2}, s_{\tilde{\tau}} \rightarrow c_{\tilde{\tau}}$ and $c_{\tilde{\tau}} \rightarrow-s_{\tilde{\tau}}$. These three groups of four graphs, 


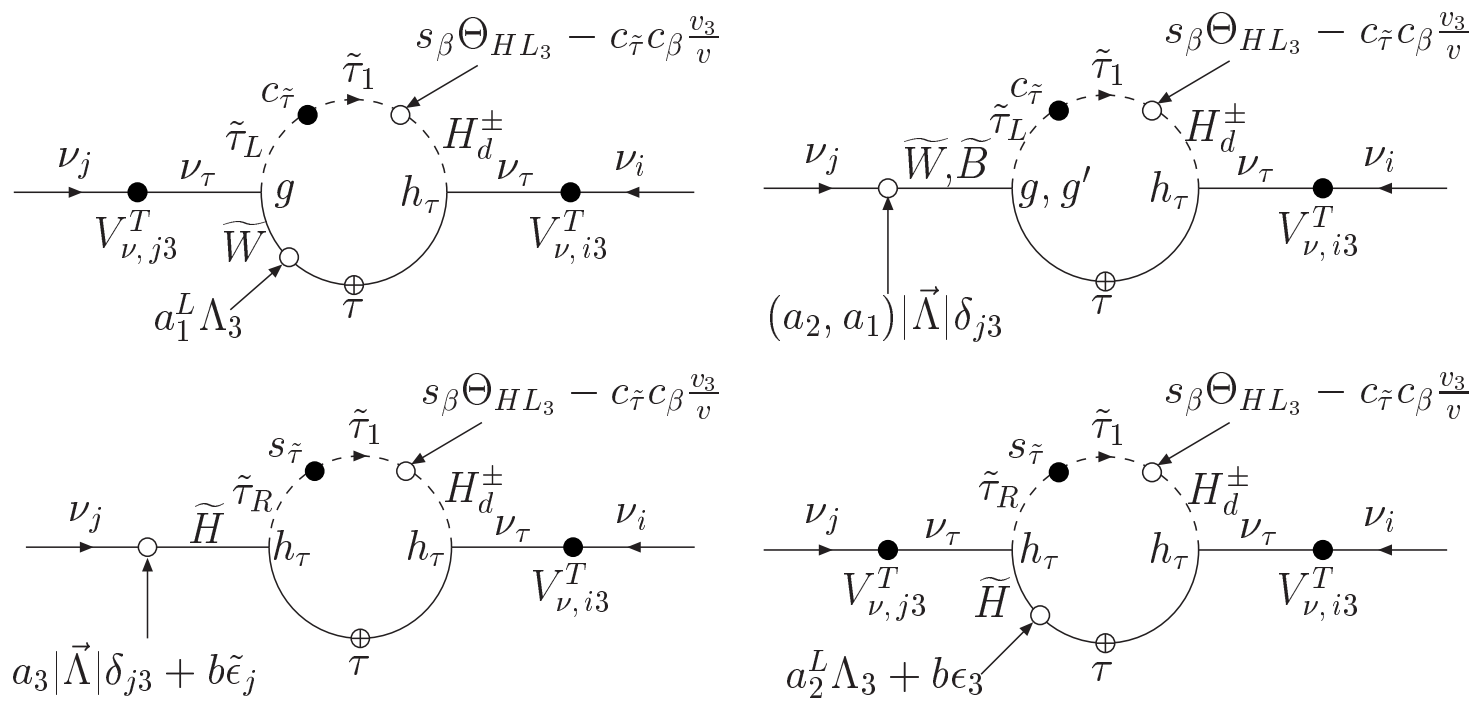

Figure 10: $\tilde{\tau}_{1}$ contributions to $\Delta B_{0}^{H^{ \pm} \tilde{\tau}_{1}}$ and $\Delta B_{0}^{G^{ \pm} \tilde{\tau}_{1} \tilde{\tau}_{2}}$

when combined, form a set which is ultraviolet finite and account for the terms in Eq. (30) proportional to $\Delta B_{0}^{H^{+} \tilde{\tau}_{1}}$ and $\Delta B_{0}^{H^{+} \tilde{\tau}_{2}}$.

C.3 $\Delta B_{0}^{H^{ \pm} L_{1}}$ and $\Delta B_{0}^{H^{ \pm} L_{2}}$
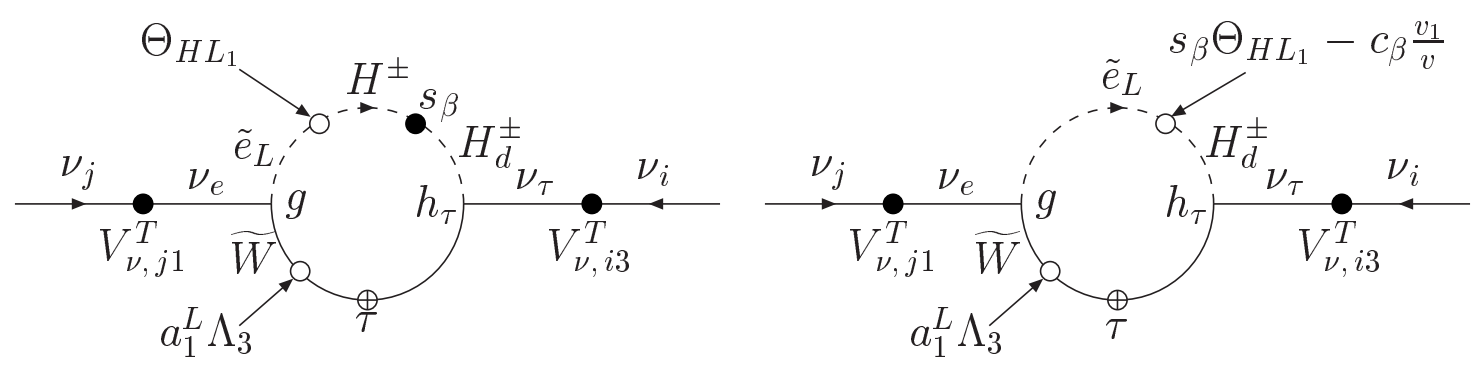

Figure 11: a) $H^{ \pm}$contribution to $\Delta B_{0}^{H^{ \pm} L_{1}}$; b) $\tilde{e}_{L}$ contribution to $\Delta B_{0}^{H^{ \pm} L_{1}}$ and $\Delta B_{0}^{G^{ \pm} L_{1}}$

We now turn our attention to the terms proportional to $\Delta B_{0}^{H^{ \pm} L_{1}}$ and $B_{0}^{H^{ \pm} L_{2}}$ which are related to the mixing between charged Higgs with selectrons and smuons. The terms proportional to $\Delta B_{0}^{H^{ \pm} L_{1}}$ come from the diagrams of Fig. 11]. The terms proportional to $\Delta B_{0}^{H^{ \pm} L_{2}}$ are easily obtained from these by replacing the corresponding slepton lines and couplings. Notice that in Fig. 11] b) there is a contribution proportional to $v_{1} / v$ that does not belong to this term. We will show below that it will contribute to the $\Delta B_{0}^{G^{ \pm} L_{1}}$ term. 
C.4 $\Delta B_{0}^{G^{ \pm} L_{1}}$ and $\Delta B_{0}^{G^{ \pm} L_{2}}$

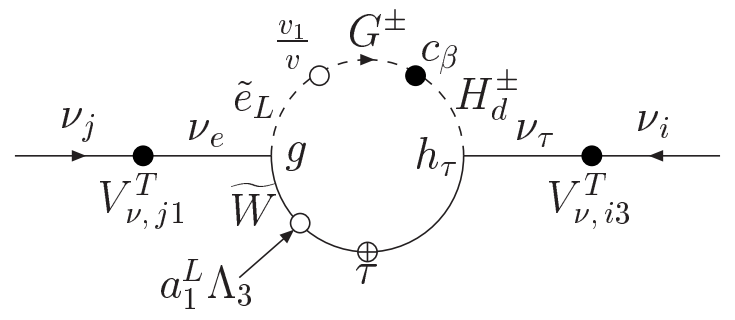

Figure 12: $G^{ \pm}$contribution to $\Delta B_{0}^{G^{ \pm} L_{1}}$

The graphs contributing to the $\Delta B_{0}^{G^{ \pm} L_{1}}$ and $\Delta B_{0}^{G^{ \pm} L_{2}}$ terms are related to those of Fig. 11. They are given by Fig. 12 and by the term proportional to $v_{1} / v$ in Fig. 111 b), for the case of the selectron. The terms proportional to $\Delta B_{0}^{G^{ \pm} L_{2}}$ are easily obtained from these by replacing the corresponding slepton lines and couplings.

\section{C.5 $\Delta B_{0}^{G^{ \pm} \tilde{\tau}_{1} \tilde{\tau}_{2}}$}

We now consider a more complicated term, the one proportional to $\Delta B_{0}^{G^{ \pm} \tilde{\tau}_{1} \tilde{\tau}_{2}}$. This term gives a finite ultraviolet contribution and comes from the diagrams of Fig. 13, together with the parts of the diagrams of Fig. 10] that are proportional to $v_{3} / v$. Corresponding to the diagrams in Fig. 13 proportional to $v_{3} / v$, there is another set with $\tilde{\tau}_{1}$ and $\tilde{\tau}_{2}$ interchanged in the usual way.
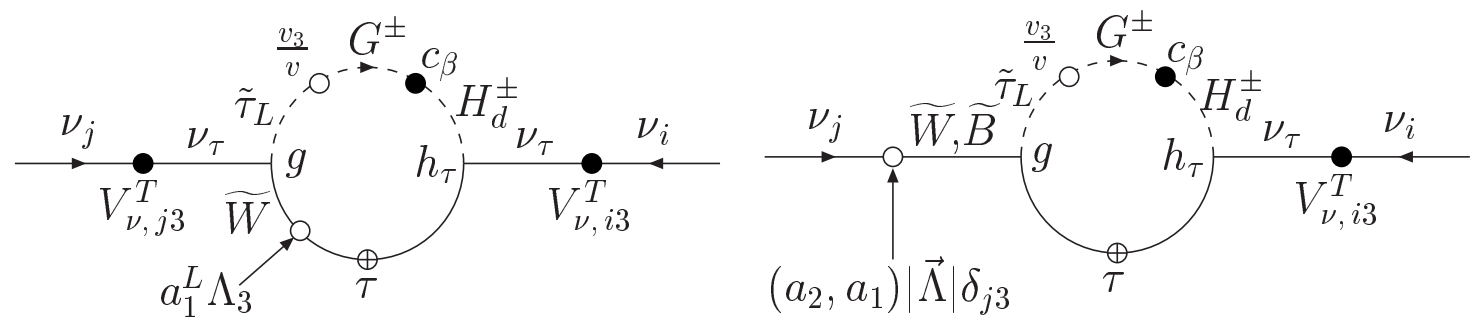

Figure 13: $G^{ \pm}$contribution to $\Delta B_{0}^{G^{ \pm} \tilde{\tau}_{1} \tilde{\tau}_{2}}$

\section{C.6 $\Delta B_{0}^{G^{ \pm} H^{ \pm} \tilde{\tau}_{1} \tilde{\tau}_{2}}$}

Let us consider finally the last term in Eq. (301), the one proportional to $\Delta B_{0}^{G^{ \pm} H^{ \pm} \tilde{\tau}_{1} \tilde{\tau}_{2}}$. This term gives an ultraviolet finite contribution and comes from four diagrams. The first 
two are those represented in Fig. 14 corresponding to a $H^{ \pm}$and $\tilde{\tau}_{1}$ propagating in the loop. The other two are obtained from these with the replacements,

$$
\begin{gathered}
H^{ \pm} \rightarrow G^{ \pm} \quad, \quad s_{\beta} \rightarrow c_{\beta} \\
\tilde{\tau}_{1} \rightarrow \tilde{\tau}_{2} \quad, \quad s_{\tilde{\tau}} \rightarrow c_{\tilde{\tau}}
\end{gathered}
$$
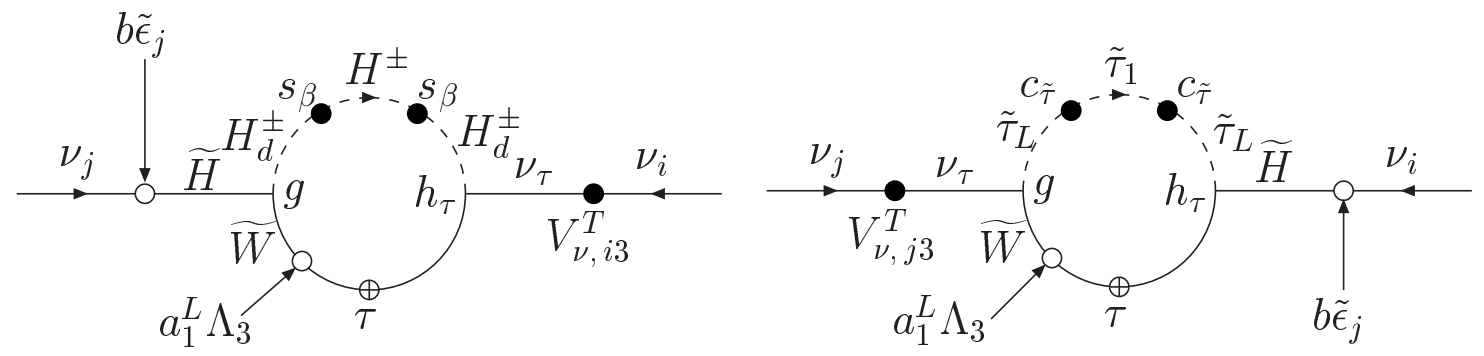

Figure 14: $H^{ \pm}$and $\tilde{\tau}_{1}$ contributions to $\Delta B_{0}^{G^{ \pm} H^{ \pm} \tilde{\tau}_{1} \tilde{\tau}_{2}}$

\section{References}

[1] Q. R. Ahmad et al. [SNO Collaboration], Phys. Rev. Lett. 89, 011301 (2002) arXiv:nucl-ex/0204008.

[2] M. Maltoni, T. Schwetz, M. A. Tortola and J. W. Valle, arXiv:hep-ph/0207227, to be published in Phys. Rev. D. This contains a full set of references on pre-KamLAND analyses of solar neutrino data. For references to relevant experimental papers see [4]

[3] K. Eguchi et al, [KamLAND Collaboration], arXiv:hep-ex/0212021.

[4] For a recent review see S. Pakvasa and J. W. Valle, arXiv:hep-ph/0301061.

[5] M. Maltoni, T. Schwetz and J. W. Valle, arXiv:hep-ph/0212129, for a full set of references on post-KamLAND analyses of solar neutrino data see [4].

[6] M. C. Gonzalez-Garcia, P. C. de Holanda, C. Pena-Garay and J. W. Valle, Nucl. Phys. B 573, 3 (2000) arXiv:hep-ph/9906469.

[7] Y. Fukuda et al. [Super-Kamiokande Collaboration], Phys. Rev. Lett. 81, 1562 (1998) arXiv:hep-ex/9807003. 
[8] M Gell-Mann, P Ramond, R. Slansky, in Supergravity, ed. P. van Niewenhuizen and D. Freedman (North Holland, 1979); T. Yanagida, in KEK lectures, ed. O. Sawada and A. Sugamoto (KEK, 1979)

[9] R. N. Mohapatra and G. Senjanovic, Phys. Rev. D 23, 165 (1981).

[10] J. Schechter and J. W. Valle, Phys. Rev. D 25, 774 (1982).

[11] G. G. Ross and J. W. Valle, Phys. Lett. B 151 (1985) 375. L. J. Hall and M. Suzuki, Nucl. Phys. B 231, 419 (1984). J. R. Ellis et al, Phys. Lett. B 150 (1985) 142. C. S. Aulakh and R. N. Mohapatra, Phys. Lett. B 119 (1982) 13 A. Santamaria and J. W. Valle, Phys. Lett. B 195, 423 (1987). Phys. Rev. Lett. 60, 397 (1988). Phys. Rev. D 39, 1780 (1989).

[12] M. A. Diaz, J. C. Romao and J. W. Valle, Nucl. Phys. B 524, 23 (1998) arXiv:hep-ph/9706315. For a review see J. W. Valle, arXiv:hep-ph/9808292.

[13] A. Bartl, W. Porod, D. Restrepo, J. Romao and J. W. Valle, Nucl. Phys. B 600 (2001) 39 arXiv:hep-ph/0007157.

[14] W. Porod, M. Hirsch, J. Romao and J. W. Valle, Phys. Rev. D 63, 115004 (2001) arXiv:hep-ph/0011248.

[15] B. Mukhopadhyaya, S. Roy and F. Vissani, Phys. Lett. B 443, 191 (1998) arXiv:hep-ph/9808265.

[16] S. Y. Choi, E. J. Chun, S. K. Kang and J. S. Lee, Phys. Rev. D 60, 075002 (1999) arXiv:hep-ph/9903465.

[17] M. Hirsch, W. Porod, J. C. Romao and J. W. Valle, Phys. Rev. D 66 (2002) 095006 arXiv:hep-ph/0207334.

[18] D. Restrepo, W. Porod and J. W. Valle, Phys. Rev. D 64, 055011 (2001) arXiv:hep-ph/0104040.

[19] B. Allanach et al., arXiv:hep-ph/9906224. 
[20] F. de Campos et al, Nucl. Phys. B 451, 3 (1995) arXiv:hep-ph/9502237. Y. Grossman and H. E. Haber, Phys. Rev. D 63, 075011 (2001) arXiv:hep-ph/0005276.

[21] T. Banks, Y. Grossman, E. Nardi and Y. Nir, Phys. Rev. D 52, 5319 (1995) arXiv:hep-ph/9505248.

[22] B. de Carlos and P. L. White, Phys. Rev. D 54, 3427 (1996) arXiv:hep-ph/9602381.

[23] A. G. Akeroyd et al, Nucl. Phys. B 529, 3 (1998) arXiv:hep-ph/9707395.

[24] F. de Campos, O. J. P. Éboli, M. A. García-Jareño and J. W. F. Valle, Nucl. Phys. B 546, 33 (1999); R. Kitano and K. Oda, Phys. Rev. D 61, 113001 (2000); D. E. Kaplan and A. E. Nelson, JHEP 0001, 033 (2000); C.-H. Chang and T.-F. Feng, Eur. Phys. J. C12, 137 (2000); M. Frank, Phys. Rev. D 62, 015006 (2000); F. Takayama and M. Yamaguchi, Phys. Lett. B 476, 116 (2000); K. Choi, E. J. Chun and K. Hwang, Phys. Lett. B 488, 145 (2000); M.A. Diaz, R.A. Lineros, M.A. Rivera, hep-ph/0210182; F. De Campos, M.A. Diaz, O.J.P. Eboli, M.B. Magro, P.G. Mercadante, Nucl. Phys. B 623, 47 (2002).

[25] A. S. Joshipura and M. Nowakowski, Phys. Rev. D 51, 2421 (1995); G. Bhattacharyya, D. Choudhury and K. Sridhar, Phys. Lett. B 349, 118 (1995); A. Yu. Smirnov and F. Vissani, Nucl. Phys. B 460, 37 (1996); J. C. Romão, F. de Campos, M. A. García-Jareño, M. B. Magro and J. W. F. Valle, Nucl. Phys. B 482, 3 (1996); R. Hempfling, Nucl. Phys. B 478, 3 (1996);

[26] M. A. Díaz, J. Ferrandis, J. C. Romão and J. W. F. Valle, Phys. Lett. B 453, 263 (1999); A. G. Akeroyd, M. A. Díaz and J. W. F. Valle, Phys. Lett. B 441, 224 (1998); M. A. Díaz, E. Torrente-Lujan and J. W. F. Valle, Nucl. Phys. B 551, 78 (1999); M. A. Díaz, J. Ferrandis, J. C. Romão and J. W. F. Valle, Nucl. Phys. B 590, 3 (2000); M. A. Díaz, D. A. Restrepo and J. W. F. Valle, Nucl. Phys. B 583, 182 (2000); M. A. Díaz, J. Ferrandis and J. W. F. Valle, Nucl. Phys. B 573, 75 (2000).

[27] S. Roy, B. Mukhopadhyaya, Phys. Rev. D 55, 7020 (1997); K. Cheung, O.C.W. Kong, Phys. Rev. D 64, 095007 (2001); T.F. Feng, X.Q. Li, Phys. Rev. D 63, 073006 
(2001); E.J. Chun, S.K. Kang, Phys. Rev. D 61, 075012 (2000); J. Ferrandis, Phys. Rev. D 60, 095012 (1999); A.G. Akeroyd, C. Liu, J. Song, Phys. Rev. D 65, 015008 (2002); D. Suematsu, Phys. Lett. B 506, 131 (2001).

[28] M. Hirsch, M. A. Diaz, W. Porod, J. C. Romao and J. W. Valle, Phys. Rev. D 62, 113008 (2000) [Erratum-ibid. D 65, 119901 (2002)] arXiv:hep-ph/0004115|; J. C. Romao, M. A. Diaz, M. Hirsch, W. Porod and J. W. Valle, Phys. Rev. D 61, 071703 (2000) arXiv:hep-ph/9907499.

[29] E. J. Chun, D. W. Jung and J. D. Park, arXiv:hep-ph/0211310, F. Borzumati and J. S. Lee, Phys. Rev. D 66 (2002) 115012 arXiv:hep-ph/0207184. E. J. Chun and J. S. Lee, Phys. Rev. D 60, 075006 (1999) arXiv:hep-ph/9811201. A. Abada, S. Davidson and M. Losada, Phys. Rev. D 65 (2002) 075010 arXiv:hep-ph/0111332.

[30] A. Masiero and J. W. Valle, Phys. Lett. B 251, 273 (1990).

[31] J. C. Romao, C. A. Santos and J. W. Valle, Phys. Lett. B 288, 311 (1992).

[32] J. C. Romao and J. W. Valle, Nucl. Phys. B 381 (1992) 87; J. C. Romao, F. de Campos and J. W. Valle, Phys. Lett. B 292 (1992) 329 arXiv:hep-ph/9207269; M. Shiraishi, I. Umemura and K. Yamamoto, Phys. Lett. B 313 (1993) 89. For bounds on spontaneous R-parity violation see P. Abreu et al. [DELPHI Collaboration], Phys. Lett. B 502 (2001) 24 arXiv:hep-ex/0102045 and D. Magalhaes Moraes, CERNTHESIS-2002-021

[33] J. M. Mira, E. Nardi, D. A. Restrepo and J. W. Valle, Phys. Lett. B 492 (2000) 81 arXiv:hep-ph/0007266.

[34] G. F. Giudice and A. Masiero, Phys. Lett. B 206, 480 (1988).

[35] H. P. Nilles and N. Polonsky, Nucl. Phys. B 484, 33 (1997) arXiv:hep-ph/9606388.

[36] M. Hirsch and J. W. Valle, Nucl. Phys. B 557, 60 (1999) arXiv:hep-ph/9812463.

[37] M. Nowakowski and A. Pilaftsis, Nucl. Phys. B 461, 19 (1996). 
[38] G. Passarino and M. J. Veltman, Nucl. Phys. B 160 (1979) 151

[39] Numerical algorithms for Passarino-Veltman functions have been published in: G. J. van Oldenborgh and J. A. Vermaseren, Z. Phys. C 46 (1990) 425; T. Hahn, M. Perez-Victoria, Comput.Phys.Commun. 118 (1999) 153, see http://www.feynarts.de/looptools/ 